\title{
LOCAL-GLOBAL PRINCIPLES FOR NORM ONE TORI OVER SEMI-GLOBAL FIELDS
}

\author{
SUMIT CHANDRA MISHRA
}

\begin{abstract}
Let $K$ be a complete discretely valued field with the residue field $\kappa$. Let $F$ be the function field of a smooth, projective, geometrically integral curve over $K$ and $\mathscr{X}$ be a regular proper model of $F$ such that the reduced special fibre $X$ is a union of regular curves with normal crossings. Suppose that the graph associated to $\mathscr{X}$ is a tree (e.g. $F=K(t))$. Let $L / F$ be a Galois extension of degree $n$ such that $n$ is coprime to $\operatorname{char}(\kappa)$. Suppose that $\kappa$ is an algebraically closed field or a finite field containing a primitive $n^{\text {th }}$ root of unity. Then we show that the local-global principle holds for the norm one torus associated to the extension $L / F$ with respect to discrete valuations on $F$ i.e. an element in $F^{\times}$is a norm from the extension $L / F$ if and only if it is a norm from the extensions $L \otimes_{F} F_{\nu} / F_{\nu}$ for all discrete valuations $\nu$ of $F$.
\end{abstract}

\section{INTRODUCTION}

Let $F$ be a field and $\Omega_{F}$ be the set of all discrete valuations on $F$. For $\nu \in \Omega_{F}$, let $F_{\nu}$ denote the completion of $F$ at $\nu$. Let $G$ be a linear algebraic group over $F$. One says that the local-global principle holds for $G$ if for any $G$-torsor $X, X$ has a rational point over $F$ if and only if it has a rational point over $F_{\nu}$ for all $\nu \in \Omega_{F}$. If $F$ is a number field, we also consider the completions at archimedean places while discussing local-global principles for algebraic groups. If $F$ is a number field, then it is known that the local-global principle holds for various classes of linear algebraic groups ([18, Chapter 6$])$, including semisimple simply connected groups. In particular, it is well-known that if $T_{L / F}$ is the norm one torus associated to a cyclic extension $L / F$, then the local-global principle holds for $T_{L / F}$ i.e. an element $\lambda \in F^{\times}$ is a norm from the extension $L / F$ if and only if $\lambda$ is a norm from $F \otimes_{F} F_{\nu} / F_{\nu}$ for all $\nu \in \Omega_{F}$ ([2, Chapter 11]). However, very little is known for general fields.

Let $K$ be a complete discretely valued field with residue field $\kappa$. Let $F$ be the function field of a smooth, projective, geometrically integral curve over $K$. Such a field $F$ is called semi-global field. Let $G$ be a linear algebraic group over $F$. Harbater, Hartmann and Krashen ([12]) developed patching techniques to study $G$-torsors over $F$ and proved that if $G$ is connected and $F$-rational, then a $G$-torsor over $F$ has a rational point over $F$ if and only if it has a rational point over certain overfields of $F$ which are defined using patching (see Subsection 2.1). As a consequence of this result, Colliot-Thélène, Parimala and Suresh ([4, Theorem 4.3.]) showed that if $G$ is reductive, $F$-rational and defined over the ring of integers of $K$, then the local-global principle holds for $G$. Similar local-global principles are proved for various linear algebraic groups $G$ over $F$ if the residue field of $K$ is either finite or algebraically closed field ([4], [5], [10], [15], [17]).

The first example of a linear algebraic group $G$ over $F$ where such a local-global principle fails was given by Colliot-Thélène, Parimala and Suresh ([5, Section 3.1. \& Proposition 5.9.]). In their example, the residue field of $K$ is the field of complex 
numbers, $G$ is the norm one torus of a Galois extension $L / F$ with Galois group $\mathbf{Z} / 2 \mathbf{Z} \times \mathbf{Z} / 2 \mathbf{Z}$ and the field $F$ has a regular proper model with the associated graph not a tree. Suppose that $F$ has a regular proper model with the associated graph a tree. If $L / F$ is a Galois extension with Galois group $\mathbf{Z} / 2 \mathbf{Z} \times \mathbf{Z} / 2 \mathbf{Z}$ and $\kappa$ is algebraically closed, then they also proved that the local-global principle holds for the norm one torus $T_{L / F}([5$, Section 3.1. \& Corollary 6.2.]).

The main aim of this paper is to prove the following theorem (see Corollary 6.4):

Theorem 1.1. Let $K$ be a complete discretely valued field with residue field $\kappa$ and $F$ be the function field of a smooth, projective, geometrically integral curve over $K$. Let $\mathscr{X}$ be a regular proper model of $F$ with reduced special fibre $X$ a union of regular curves with normal crossings. Let $L / F$ be a Galois extension over $F$ of degree $n$ with Galois group $G$. Suppose that the graph associated to $\mathscr{X}$ is a tree and $\kappa$ is one of the following:

- $\kappa$ is an algebraically closed field of characteristic coprime to $n$, or

- $\kappa$ is a finite field of characteristic coprime to $n$ and contains a primitive $n^{\text {th }}$ root of unity.

Then the local-global principle holds for the norm one torus $T_{L / F}$ i.e. an element $\lambda \in F$ is a norm from the extension $L / F$ if and only if $\lambda$ is a norm from the extensions $L \otimes_{F} F_{\nu} / F_{\nu}$ for all $\nu \in \Omega_{F}$.

For a finite separable extension $L / F$, let $T_{L / F}$ denote the norm one torus associated to $L / F$. For any extension $N / F$, let $R T_{L / F}(N)$ be the subgroup of $T_{L / F}(N)$ consisting of $R$-trivial elements (see Subsection 2.3). The above theorem follows from the following more general theorem (6.3), where we allow more general residue fields $\kappa$ :

Theorem 1.2. Let $K$ be a complete discretely valued field with residue field $\kappa$ and $F$ be the function field of a smooth, projective, geometrically integral curve over $K$. Let $\mathscr{X}_{0}$ be a regular proper model of $F$ with reduced special fibre $X_{0}$ a union of regular curves with normal crossings. Let $L / F$ be a Galois extension over $F$ of degree $n$. Suppose that the graph associated to $\mathscr{X}_{0}$ is a tree and:

- $\operatorname{char}(\kappa)$ is coprime to $n$,

- $\kappa$ contains a primitive $n^{\text {th }}$ root of unity $\rho$, and

- for all finite extensions $\kappa^{\prime} / \kappa$ and for all finite Galois extensions $l / \kappa^{\prime}$ of degree $d$ dividing $n$,

$$
T_{l / \kappa^{\prime}}\left(\kappa^{\prime}\right)=R T_{l / \kappa^{\prime}}\left(\kappa^{\prime}\right)<\rho^{\frac{n}{d}}>
$$

Then the local-global principle holds for the norm one torus $T_{L / F}$. 
Remark: In fact one can restrict to divisorial discrete valuations in the above theorems.

In the last section of the paper, we also give counterexamples to the local-global principle for certain norm one tori and multinorm tori over a semi-global field.

We now briefly describe the strategy of the proof of Theorem 1.2. Let $K$ be a complete discretely valued field with residue field $\kappa$ and $F$ be the function field of a smooth, projective, geometrically integral curve over $K$. Let $\mathscr{X}$ be a regular proper model of $F$ with reduced special fibre $X$ a union of regular curves with normal crossings. For any point $P \in X$, let $F_{P}$ be the fraction field of the completion of the local ring at $P$ on $\mathscr{X}$.

For a linear algebraic group $G$ over $F$, let us define:

$$
\begin{gathered}
\bullet \amalg_{X}(F, G):=\operatorname{ker}\left(H^{1}(F, G) \rightarrow \prod_{P \in X} H^{1}\left(F_{P}, G\right)\right) \\
\bullet \amalg(F, G):=\operatorname{ker}\left(H^{1}(F, G) \rightarrow \prod_{\nu \in \Omega_{F}} H^{1}\left(F_{\nu}, G\right)\right) .
\end{gathered}
$$

It is known that $\amalg_{X}(F, G) \subseteq \amalg(F, G)([12$, Proposition 8.2.]).

In general, $\amalg_{X}(F, G)$ and $\amalg(F, G)$ are just pointed sets, but they are abelian groups if $G$ is abelian. The pointed sets $\amalg_{X}(F, G)$ and $\amalg(F, G)$ measure the obstruction to the local-global principle for the group $G$ with respect to points on $X$ and with respect to discrete valuations on $F$ respectively.

First we prove the following (6.2):

Theorem 1.3. Let $K$ be a complete discretely valued field with residue field $\kappa$ and $F$ be the function field of a smooth, projective, geometrically integral curve over $K$. Let $\mathscr{X}$ be a regular proper model of $F$ with reduced special fibre $X$ a union of regular curves with normal crossings. Let $L / F$ be a Galois extension over $F$ of degree $n$. Suppose that

- $n$ is coprime to $\operatorname{char}(\kappa)$,

- $\kappa$ contains a primitive $n^{\text {th }}$ root of unity $\rho$,

- for all finite extensions $\kappa^{\prime} / \kappa$ and for all finite Galois extensions $l / \kappa^{\prime}$ of degree $d$ dividing $n$,

$$
T_{l / \kappa^{\prime}}\left(\kappa^{\prime}\right)=R T_{l / \kappa^{\prime}}\left(\kappa^{\prime}\right)<\rho^{\frac{n}{d}}>
$$

- the graph associated to $\mathscr{X}$ is a tree.

Then $\amalg_{X}\left(F, T_{L / F}\right)=0$. 
We conclude our main theorem (Theorem 1.2), by proving that for $K, F$ and $L$ as in Theorem 1.3, $\amalg\left(F, T_{L / F}\right)=\bigcup_{X} \amalg_{X}(F, G)(5.1)$, where $X$ is running over the reduced special fibres of regular proper models $\mathscr{X}$ of $F$ which are obtained as a sequence of blow-ups of $\mathscr{X}_{0}$ centered at closed points of $\mathscr{X}_{0}$.

\section{Preliminaries}

In this section, we recall a few basic definitions and facts about patching and $R$ equivalence on algebraic groups ([3], [8], [11], [12]) which will be used in this paper.

\subsection{Patching and various Shas.}

Let $F$ be a field and $\Omega_{F}$ be the set of all equivalence classes of discrete valuations $\nu$ on $F$. For $\nu \in \Omega_{F}$, let $F_{\nu}$ denote the completion of $F$ at $\nu$ and $\kappa(\nu)$ the residue field at $\nu$. For an algebraic group $G$ over $F$, let

$$
\amalg(F, G):=\operatorname{ker}\left(H^{1}(F, G) \rightarrow \prod_{\nu \in \Omega_{F}} H^{1}\left(F_{\nu}, G\right)\right) .
$$

In this paper, we are concerned with a special class of fields called semi-global fields.

Definition 2.1. (Semi-global field) A semi-global field is the function field of a smooth, projective, geometrically integral curve over a complete discretely valued field.

Example: Some examples of semi-global fields are: $\mathbb{C}((t))(x), \mathbb{Q}_{p}(x), \mathbb{F}_{p}((t))(x)$ and $\frac{\mathbb{C}((t))(x)[y]}{\langle x y(x+y-1)-t\rangle}$.

Let $T$ be a complete discretely valued ring with fraction field $K$ and residue field $\kappa$. Let $t \in T$ be a parameter. Let $F$ be a function field of a smooth, projective, geometrically integral curve over $K$. Thus $F$ is a semi-global field. Then there exists a regular 2-dimensional integral scheme $\mathscr{X}$ which is proper over $T$ with function field $F$. We call such a scheme $\mathscr{X}$ a regular proper model of $F$. Further there exists a regular proper model of $F$ with the reduced special fibre a union of regular curves with only normal crossings. Let $\mathscr{X}$ be a regular proper model of $F$ with the reduced special fibre $X$ a union of regular curves with only normal crossings.

For a semi-global field $F$ and a regular proper model $\mathscr{X}$ of $F$, one can associate three different kinds of overfields of $F$. We describe them below and discuss how they are related to each other.

For any point $x$ of $\mathscr{X}$, let $R_{x}$ be the local ring at $x$ on $\mathscr{X}, \widehat{R}_{x}$ the completion of the local ring $R_{x}, F_{x}$ the fraction field of $\widehat{R}_{x}$ and $\kappa(x)$ the residue field at $x$.

For any subset $U$ of $X$ that is contained in an irreducible component of $X$, let $R_{U}$ be the subring of $F$ consisting of the rational functions which are regular at every point of $U$. Let $\widehat{R_{U}}$ be the $t$-adic completion of $R_{U}$ and $F_{U}$ the fraction field of $\widehat{R_{U}}$.

Let $\eta \in X$ be a codimension zero point and $P \in X$ a closed point such that $P$ is in the closure $X_{\eta}$ of $\eta$. Such a pair $(P, \eta)$ is called a branch. For a branch $(P, \eta)$, we define $F_{P, \eta}$ to be the completion of $F_{P}$ at the discrete valuation of $F_{P}$ associated to $\eta$. We call such fields branch fields. If $\eta$ is a codimension zero point of $X, U \subset X_{\eta}$ 
an open subset and $P \in X_{\eta}$ a closed point, then we will use $(P, U)$ to denote the branch $(P, \eta)$ and $F_{P, U}$ to denote the field $F_{P, \eta}$.

With $P, U, \eta$ as above, there are natural inclusions of $F_{P}, F_{U}$ and $F_{\eta}$ into $F_{P, \eta}=$ $F_{P, U}$. Also, there is a natural inclusion of $F_{U}$ into $F_{\eta}$.

Let $\mathcal{P}$ be a non-empty finite set of closed points of $X$ that contains all the closed points of $X$ where distinct irreducible components of $X$ meet. Let $\mathcal{U}$ be the set of connected components of the complement of $\mathcal{P}$ in $X$ and let $\mathcal{B}$ be the set of branches $(P, U)$ with $P \in \mathcal{P}$ and $U \in \mathcal{U}$ with $P$ in the closure of $U$.

Let $G$ be a linear algebraic group over $F$. Let us define

$$
\amalg_{\mathscr{X}, \mathcal{P}}(F, G):=\operatorname{ker}\left(H^{1}(F, G) \rightarrow \prod_{\xi \in \mathcal{P} \cup \mathcal{U}} H^{1}\left(F_{\xi}, G\right)\right) .
$$

If $\mathscr{X}$ is understood, we will just use the notation $\amalg_{\mathcal{P}}(F, G)$.

Similarly, let us define

$$
\amalg_{\mathscr{X}, X}(F, G):=\operatorname{ker}\left(H^{1}(F, G) \rightarrow \prod_{P \in X} H^{1}\left(F_{P}, G\right)\right) .
$$

Again, if $\mathscr{X}$ is understood, we will just use the notation $\amalg_{X}(F, G)$.

We have a bijection([12, Corollary 3.6.]):

$$
\prod_{U \in \mathcal{U}} G\left(F_{U}\right) \backslash \prod_{(P, U) \in \mathcal{B}} G\left(F_{P, U}\right) / \prod_{P \in \mathcal{P}} G\left(F_{P}\right) \rightarrow \amalg_{\mathcal{P}}(F, G)
$$

This is a very useful result since the double coset mentioned above is usually more manageable to work with.

By $\left(\left[12\right.\right.$, Corollary 5.9.]), we have $\amalg_{X}(F, G)=\bigcup \amalg_{\mathcal{P}}(F, G)$ where union ranges over all finite subsets $\mathcal{P}$ of closed points of $\mathscr{X}$ which contain all the singular points of $X$. We also have $\amalg_{X}(F, G) \subseteq \amalg(F, G)$ ([12, Proposition 8.2.]).

2.2. The associated graph. We start with a basic fact about finite bipartite trees:

Lemma 2.2. Let $\Gamma$ be a finite bipartite graph and $G$ be an abstract group. Let $V$ be the set of vertices with parts $V_{1}$ and $V_{2}$. For each edge $\theta$ of $\Gamma$, let $g_{\theta} \in G$. If $\Gamma$ is a tree, then for every $v \in V$, there exists $g_{v} \in G$ such that if $e$ is an edge joining two vertices $v_{i} \in V_{i}$, then $g_{e}=g_{v_{1}} g_{v_{2}}$.

Proof. Suppose that $\Gamma$ is a tree. Without loss of generality, we may assume that $\Gamma$ is a connected graph. We prove the lemma by the induction on number of vertices. Suppose that $\Gamma$ has one one vertex. Then there is nothing to prove.

Suppose that $\Gamma$ has more than one vertex. Since $\Gamma$ is a connected tree, there exists a vertex $v_{0} \in V$ with exactly one edge $\theta$ at $v_{0}$. Without loss of generality, we may assume $v_{0} \in V_{1}$. Let $\Gamma^{\prime}$ be the graph obtained from $\Gamma$ by removing the vertex $v_{0}$ and the edge $\theta$. Then $\Gamma^{\prime}$ is again a finite bipartite graph which is a tree. Then, by induction hypothesis, for every vertex $v$ of $\Gamma^{\prime}$, there exists $g_{v} \in G$ such that if $e$ is an edge in $\Gamma^{\prime}$ joining $v_{1} \in V_{1} \backslash\left\{v_{0}\right\}$ and $v_{2} \in V_{2}$, then $g_{e}=g_{v_{1}} g_{v_{2}}$. Let $v_{0}^{\prime} \in V_{2}$ be the 
other vertex of the edge $\theta$. Let $g_{v_{0}}=g_{\theta} g_{v_{0}^{\prime}}^{-1}$. Then it follows that $g_{v}$ have the required property.

Let $T$ be a complete discretely valued ring with fraction field $K$, and residue field $\kappa$. Let $t \in T$ be a parameter. Let $F$ be the function field of a smooth, projective, geometrically integral curve over $K$ and $\mathscr{X}$ be a regular proper model of $F$ with the reduced special fibre $X$ a union of regular curves with only normal crossings. Let $\mathcal{P}$ be a non-empty finite set of closed points of $X$ that contains all the closed points of $X$ where distinct irreducible components of $X$ meet. Let $\mathcal{U}$ be the set of connected components of the complement of $\mathcal{P}$ in $X$ and let $\mathcal{B}$ be the set of branches $(P, U)$ with $P \in \mathcal{P}$ and $U \in \mathcal{U}$ with $P$ in the closure of $U$.

We have a graph $\Gamma(\mathscr{X}, \mathcal{P})$ associated to $\mathscr{X}$ and $\mathcal{P}$ whose vertices are elements of $\mathcal{P} \cup \mathcal{U}$ and edges are elements of $\mathcal{B}$. Since there are no edges between any vertices which are in $\mathcal{P}$ (resp. $\mathcal{U}), \Gamma(\mathscr{X}, \mathcal{P})$ is a finite bipartite graph with parts $\mathcal{P}$ and $\mathcal{U}$. If $\mathcal{P}^{\prime}$ is another finite set of closed points of $X$ containing all the closed points of $X$ where distinct irreducible components of $X$ meet, then $\Gamma(\mathscr{X}, \mathcal{P})$ is a tree is and only if $\Gamma\left(\mathscr{X}, \mathcal{P}^{\prime}\right)$ is a tree $([12$, Remark $6.1(\mathrm{~b})])$. Hence if $\Gamma(\mathscr{X}, \mathcal{P})$ is a tree for some $\mathcal{P}$ as above, then we say that the graph $\Gamma(\mathscr{X})$ associated to $\mathscr{X}$ is a tree.

Now we have the following result as a corollary to Lemma 2.2:

Corollary 2.3. Let $F, \mathscr{X}, X, \mathcal{P}, \mathcal{U}$ and $\mathcal{B}$ be as above. Let $G$ be an abstract group and for each branch $b \in \mathcal{B}$, let $g_{b} \in G$. Suppose that the graph $\Gamma(\mathscr{X})$ associated to $\mathscr{X}$ is a tree. Then for every $\zeta \in \mathcal{U} \cup \mathcal{P}$, there exists $g_{\zeta} \in G$ such that if $b=(P, U) \in \mathcal{B}$, then $g_{b}=g_{P} g_{U}$.

\subsection{R-equivalence and R-trivial elements.}

Other than patching, we use the notion of $R$-equivalence in this paper. We define the notion below and discuss results about $R$-equivalence for norm one tori, which will be used later.

Notation 2.4. Let $F$ be a field and $L$ be an étale algeba over $F$. Throughout this paper, we will denote the norm 1 torus $R_{L / F}^{1} \mathbb{G}_{m}$ by $T_{L / F}$.

Let $X$ be a variety over a field $F$. For a field extension $L$ of $F$, let $X(L)$ be the set of $L$-points of $X$. We say that two points $x_{0}, x_{1} \in X(L)$ are elementary $R$ equivalent, denoted by $x_{0} \sim x_{1}$, if there is a rational map $f: \mathbb{P}^{1}(L) \rightarrow X(L)$ such that $f(0)=x_{0}$ and $f(1)=x_{1}$. The equivalence relation generated by $\sim$ is called $R$-equivalence. When $X=G$ is an algebraic group defined over $F$ with the identity element $e$, we define $R G(L)=\{x \in G(L): x$ is $R$-equivalent to $e\}$. The elements of $R G(L)$ are called $R$-trivial elements. It is well-known that $R G(L)$ is a normal subgroup of $G(L)$ (cf. [8, p-1]) Sometimes, we denote $G(L) / R G(L)$ by $G(L) / R$. Let $L / F$ be a Galois extension with Galois group $G$, and $T_{L / F}$ the norm 1 torus associated to the extension $L / F$. Then for any extension $N / F, R T_{L / F}(N)$ is the subgroup generated by the set $\left\{a^{-1} \sigma(a): a \in\left(L \otimes_{F} N\right)^{\times}, \sigma \in G\right\}$ ([3, Proposition 15]).

Now we discuss few basic results about $R$-equivalence on norm one tori: 
Proposition 2.5. Let $F$ be a field and $L_{0} / F$ a finite extension. Let $L$ be the product of $r$ copies of $L_{0}$. Then the homomorphism $T_{L / F} \rightarrow T_{L_{0} / F}$ given by $\left(a_{1}, \cdots, a_{r}\right) \mapsto$ $a_{1} \cdots a_{r}$ induces an isomorphism $T_{L / F}(F) / R T_{L / F}(F) \rightarrow T_{L_{0} / F}(F) / R T_{L_{0} / F}(F)$.

Proof. In fact the isomorphism $\left(R_{L_{0} / F}\left(\mathbb{G}_{m}\right)\right)^{r} \rightarrow\left(R_{L_{0} / F}\left(\mathbb{G}_{m}\right)\right)^{r}$ given by sending $\left(b_{1}, \cdots, b_{r}\right)$ to $\left(b_{1}, \cdots, b_{r-1}, b_{1} b_{2} \cdots b_{r}\right)$ induces an isomorphism of algebraic groups $T_{L / F} \rightarrow\left(R_{L_{0} / F}\left(\mathbb{G}_{m}\right)\right)^{r-1} \times T_{L_{0} / F}\left(\left[7\right.\right.$, Lemma 1.1.]). Since $R_{L_{0} / F}\left(\mathbb{G}_{m}\right)$ is rational, it is $R$-trivial by ([8, Corollary 1.6.]). Hence the homomorphism $T_{L / F} \rightarrow T_{L_{0} / F}$ given by $\left(a_{1}, \cdots, a_{r}\right) \mapsto a_{1} \cdots a_{r}$ induces an isomorphism $T_{L / F}(F) / R T_{L / F}(F) \rightarrow$ $T_{L_{0} / F}(F) / R T_{L_{0} / F}(F)$.

Corollary 2.6. Let $F$ be a field and $L_{0} / F$ a finite extension of degree $d$ and $L$ the product of $r$ copies of $L_{0}$. Suppose that $F$ contains $\rho$, a primitive $(d r)^{\text {th }}$ root of unity. If $T_{L_{0} / F}(F)=R T_{L_{0} / F}(F)<\rho^{r}>$, then $T_{L / F}(F)=R T_{L / F}(F)<\rho>$.

Proof. Since $(\rho, \rho, \cdots, \rho)$ maps to $\rho^{r}$ under the isomorphism given in $(2.5)$, the corollary follows from $(2.5)$.

Lemma 2.7. Let $L / F$ be a finite Galois extension of degree $m$ and $N / F$ any field extension. If $\alpha \in\left(L \otimes_{F} N\right)^{\times}$, then $N_{L \otimes_{F} N / N}(\alpha)^{-1} \alpha^{m} \in R T_{L / F}(N)$.

Proof. Let $G$ be the Galois group of $L / F$. Since $N_{L \otimes_{F} N / N}(\alpha)=\prod_{\sigma \in G} \sigma(\alpha)$, we have

$$
N_{L \otimes_{F} N / N}(\alpha)^{-1} \alpha^{m}=\frac{\alpha^{m}}{\prod_{\sigma \in G} \sigma(\alpha)}=\prod_{\sigma \in G} \frac{\alpha}{\sigma(\alpha)} .
$$

Hence $N_{L \otimes_{F} N / N}(\alpha)^{-1} \alpha^{m} \in R T_{L / F}(N)$.

\section{Norm one Elements - COMPlete DiscRetely VAlued FIELDS}

Let $F$ be a complete discretely valued field with residue field $\kappa$. Let $L / F$ be a Galois extension. Let $l$ denote the residue field of $L$. In this section, we investigate the relationship between the groups $T_{L / F}(F) / R T_{L / F}(F)$ and $T_{l / \kappa}(\kappa) / R T_{l / \kappa}(\kappa)$. This allows us to transfer our assumptions from the residue fields to the branch fields (see Subsection 2.1), which is crucial for proving local-global principles for norm one tori for semi-global fields (see Lemma 6.1).

Lemma 3.1. Let $F$ be a complete discretely valued field with residue field $\kappa$ and $L / F$ be a finite Galois extension of degree $n$ with residue field $l$. Suppose that $n$ is coprime to char $(\kappa)$. Let $z \in T_{L / F}(F)$. If the image of $z$ in $l$ is 1 , then $z \in R T_{L / F}(F)$.

Proof. Let $S$ be the integral closure of $R$ in $L$. Then $S$ is a complete discrete valuation ring with residue field $l$. Let $z \in T_{L / F}(F)$ with the image of $z$ in $l$ is 1 . Since $n$ is coprime to $\operatorname{char}(\kappa)$, by Hensel's lemma, there is a $w \in S$ with $\bar{w}=1$ and $z=w^{n}$. Since $N_{L / F}(z)=1, N_{L / F}(w)^{n}=1$ and hence $\rho=N_{L / F}(w)$ is an $n^{\text {th }}$ root of unity. Since $\bar{w}=1, \overline{N_{L / F}(w)}=N_{l / \kappa}(\bar{w})^{e}=1$, where $e$ is the ramification index of the extension $L / F$. Hence $\bar{\rho}=1$. Since $n$ is coprime to char $(\kappa)$, by Hensel's lemma, the quotient map $S \rightarrow l$ induces a bijection from the set of $n^{\text {th }}$ roots of unity in $S$ to the set of $n^{\text {th }}$ roots of unity in $l$. Hence $\rho=1$ and $w \in T_{L / F}(F)$. Since $z=w^{n}$, $z \in R T_{L / F}(F)$ by $(2.7)$. 
Lemma 3.2. Let $F$ be a complete discretely valued field with residue field $\kappa$. Let $L / F$ be a Galois extension of degree $n$. Suppose that $(n, \operatorname{char}(\kappa))=1$. Suppose that $F$ contains a primitive $n^{\text {th }}$ root of unity $\rho_{n}$. Let $l$ be the residue field of $L$ and $f=[l: \kappa]$. If $T_{l / \kappa}(\kappa)=R T_{l / \kappa}(\kappa)<\rho_{n}^{n / f}>$, then $T_{L / F}(F)=R T_{L / F}(F)<\rho_{n}>$.

Proof. Let $R$ be the discrete valuation ring of $F$ and $S$ be the integral closure of $R$ in $L$. Let $e$ be the ramification index of the extension $L / F$. Then $n=e f$. For any element $y \in S$ (resp. $R$ ), we will use $\bar{y}$ to denote its image in the residue field $l$ (resp. $\kappa)$.

Let $x \in L$ with $N_{L / F}(x)=1$. Then, $N_{l / \kappa}(\bar{x})^{e}=\overline{N_{L / F}(x)}=1$. Hence $N_{l / \kappa}(\bar{x})=\rho_{n}^{f i}$ for some $i$ with $0 \leq i<e$. Let $y=\rho_{n}^{-i} x$. Then $N_{L / F}(y)=1$ and $N_{l / \kappa}(\bar{y})=$ $N_{l / \kappa}\left(\rho_{n}^{-i}\right) N_{l / \kappa}(\bar{x})=\rho_{n}^{-f i} \rho_{n}^{f i}=1$. Thus $\bar{y} \in T_{l / \kappa}(\kappa)$ and hence, by the assumption, $\bar{y}=\theta \rho_{n}^{e j}$ for some $\theta \in R T_{l / \kappa}(\kappa)$ and $j$ an integer. Write

$$
\theta=\prod_{\sigma \in \operatorname{Gal}(l / \kappa)}\left(a_{\sigma}\right)^{-1} \sigma\left(a_{\sigma}\right)
$$

for some $a_{\sigma} \in l^{\times}$. Since $\operatorname{Gal}(l / \kappa)$ is a quotient of $\operatorname{Gal}(L / F)$, for every $\sigma \in \operatorname{Gal}(l / \kappa)$ we choose a lift $\tilde{\sigma} \in \operatorname{Gal}(L / F)$ of $\sigma$. Let $b_{\sigma} \in S$ with $\bar{b}_{\sigma}=a_{\sigma}$ and

$$
z=y^{-1} \rho_{n}^{e j} \prod_{\sigma \in \operatorname{Gal}(l / \kappa)}\left(b_{\sigma}\right)^{-1} \tilde{\sigma}\left(b_{\sigma}\right) .
$$

Then $z \in T_{L / F}(F)$ and $\bar{z}=1$. Thus, by (3.1), $z \in R T_{L / F}(F)$. Therefore $y \in$ $R T_{L / F}(F)<\rho_{n}>$ and hence $x \in R T_{L / F}(F)<\rho_{n}>$.

Definition 3.3. A complete discretely valued field $K$ with finite residue field is called a 1-local field. For $m \geq 1$, a complete discretely valued field $K$ with $m$-local residue field $k$ is called a $(m+1)$-local field. If $K$ is a 1-local field, the residue field of $K$ is called the first residue field of $K$. If $K$ is a $(m+1)$-local field with residue field $k$, then the first residue field of $k$ is called the first residue field of $K$.

Corollary 3.4. Let $K$ be an $m$-local field with first residue field $\kappa$ or an iterated Laurent series in $m$ variables over an algebraically closed field $\kappa$. Let $L / K$ be a finite Galois extension of degree $n$. If $n$ is coprime to char $(\kappa)$ and $K$ contains a primitive $n^{\text {th }}$ root of unity $\rho_{n}$, then $T_{L / K}(K)=R T_{L / K}(K)<\rho_{n}>$.

Proof. Every finite extension $l / \kappa$ is cyclic and by Hilbert 90, $T_{l / \kappa}(\kappa)=R T_{l / \kappa}(\kappa)$. Thus, by $(3.2), T_{L / K}(F)=R T_{L / K}(K)<\rho_{n}>$. The corollary follows by induction on $m$ and by $(3.2)$.

\section{Two Dimensional COMPlete FieldS}

Let $F$ be a field with a discrete valuation $v$. Let $\kappa(v)$ be the residue field of $v$. Let $L / F$ be a finite extension and $w$ be a discrete valuation on $L$ extending $v$. Let $e(w / v)$ be the ramification index of $w$ over $v$. For any field $E, a \in E^{\times}$and $n \geq 1$, let $E(\sqrt[n]{a})$ denote the field generated by $E$ and $\sqrt[n]{a}$ in a fixed algebraic closure of $E$. 
Lemma 4.1. Let $F$ be a field with a discrete valuation $v, \pi \in F^{\times}$with $v(\pi)=1$. Let $L / F$ be a finite extension of degree coprime to char $(\kappa(v))$ and $w$ be a discrete valuation of $L$ extending $v$. Let $\ell$ be a prime not equal to char $(\kappa(v))$. Then there is a unique discrete valuation $\tilde{v}$ on $F(\sqrt[\ell]{\pi})$ extending $v$. Let $\tilde{w}$ on $L(\sqrt[\ell]{\pi})$ be a discrete valuation extending $w$. If $\ell$ divides $e(w / v)$, then $e(\tilde{w} / \tilde{v})=e(w / v) / \ell$.

Proof. Since $v(\pi)=1, v$ is totally ramified in $F(\sqrt[\ell]{\pi})$ and hence there is a unique extension $\tilde{v}$ of $v$ to $F(\sqrt[\ell]{\pi})$.

For the ramification index calculations, we can replace $F$ by $F_{v}$, the completion of $F$ with respect to the valuation $v$ and hence may assume that $F$ is complete. Let $L^{n r}$ be the maximal unramified subextension of $L / F$. Since the ramification index of $L / L^{n r}$ is same as the ramification index of $L / F$, replacing $F$ by $L^{n r}$, we may assume that $L / F$ is totally ramified. Since $n=e=[L: F]$ is coprime to $\operatorname{char}(\kappa(v))$, we have $L=F(\sqrt[n]{u \pi})$ for some $u \in F$ with $v(u)=0([2$, Proposition $1, \mathrm{p}-32])$.

By hypothesis, we have that $\ell$ divides $n$. Suppose that $u \in F^{\times \ell}$. Then $F(\sqrt[\ell]{\pi}) \subseteq L$ and hence $L / F(\sqrt[\ell]{\pi})$ is a totally ramified extension of degree $n / \ell$. Now suppose that $u \notin F^{\times \ell}$. Then $L(\sqrt[\ell]{\pi})=F(\sqrt[\ell]{\pi})(\sqrt[\ell]{u})(\sqrt[n / \ell]{\sqrt[\ell]{u \pi}})$. Hence the ramification index of the extension $L(\sqrt[\ell]{\pi}) / F(\sqrt[\ell]{\pi})$ is $n / \ell$.

Notation 4.2. Let $A$ be a complete regular local ring of dimension 2 with residue field $\kappa$ and fraction field $F$. Let $\mathfrak{m}=\left(\pi_{1}, \pi_{2}\right) \subset A$ be the maximal ideal of $A$. Then, for $i=1,2$, we denote by $\widehat{A}_{\left(\pi_{i}\right)}$ be the completion of the local ring $A_{\left(\pi_{i}\right)}$ with respect to the ideal $\left(\pi_{i}\right)$ and by $F_{\pi_{i}}$ the fraction field of $\widehat{A}_{\left(\pi_{i}\right)}$.

We are studying these fields since the fields $F_{P}$ appearing in the patching setup (see Subsection 2.1) are fraction fields of complete regular local rings of dimension 2 and the branch fields are obtained as completions of the fields $F_{P}$ as discussed above.

4.1. Structure of extensions of two dimensional complete fields. Let $F$ be as in Notation 4.2. In this subsection, we study Galois extensions of $F$.

In the following lemma, we prove that with some assumptions, a field extension $L$ over $F$ remains a field after base change to the completion $F_{\pi_{i}}$ for $i=1,2$ :

Lemma 4.3. Let $A$ be a complete regular local ring of dimension 2 with residue field $\kappa$ and fraction field $F$. Let $L / F$ be a field extension of degree $n$ where $n$ is coprime to $\operatorname{char}(\kappa)$. Let $\mathfrak{m}=\left(\pi_{1}, \pi_{2}\right) \subset A$ be the maximal ideal of $A$. Suppose that $L / F$ is unramified on $A$ except possibly at $\pi_{1}$ and $\pi_{2} \in A$. Then $L \otimes_{F} F_{\pi_{i}}$ is a field for $i=1,2$.

Proof. Let $v_{i}$ be the discrete valuation of $F$ given by $\pi_{i}$, for $i=1,2$. To show that $L \otimes_{F} F_{\pi_{i}}$ is a field, it is enough to show that there is a unique extension of $v_{i}$ to a discrete valuation on $L$.

Let $w_{i}^{j}$ be the extensions of the valuations $v_{i}$ to $L$. Let $m$ be the maximum of $e\left(w_{i}^{j_{i}} / v_{i}\right)$ for $i=1,2$; where $1 \leq j_{i} \leq n_{i}$ for some positive integers $n_{1}$ and $n_{2}$. Since each $e\left(w_{i}^{j_{i}} / v_{i}\right) \geq 1, m \geq 1$. We prove the result by induction on $m$.

Suppose that $m=1$. Then $e\left(w_{i}^{j_{i}} / v_{i}\right)=1$ for for $i=1,2$ and $1 \leq j_{i} \leq n_{i}$. Hence $L / F$ is unramified at $\pi_{i}$ for $i=1,2$. Since $L / F$ is unramified on $A$ except possibly 
at $\pi_{1}, \pi_{2}, L / F$ is unramified on $A$. Let $\tilde{A}$ be the integral closure of $A$ in $L$. Then $\tilde{A}$ is again a complete regular local ring of dimension 2 with $\left(\pi_{1}, \pi_{2}\right)$ as maximal ideal and fraction field $L$. Thus $\pi_{i}$ remains a prime over $\tilde{A}$. Hence there is a unique extension of $v_{i}$ to a discrete valuation of $L$. Hence $L \otimes_{F} F_{\pi_{i}} \cong L_{\pi_{i}}$ is a field.

Now suppose that $m>1$. Let $\ell$ be a prime which divides $m$. Let $E=F\left(\sqrt[\ell]{\pi_{1}}, \sqrt[\ell]{\pi_{2}}\right)$ and $M=L\left(\sqrt[\ell]{\pi_{1}}, \sqrt[\ell]{\pi_{2}}\right)$. Let $B$ be the integral closure of $A$ in $E$. Then, by $([16$, Corollary 3.3.]), $B$ is a regular local ring with maximal ideal $\left(\pi_{1}^{\prime}, \pi_{2}^{\prime}\right)$; where $\pi_{1}^{\prime}=\sqrt[\ell]{\pi_{1}}$ and $\pi_{2}^{\prime}=\sqrt[\ell]{\pi_{2}}$. Then $M / E$ is unramified on $B$ except possibly at $\pi_{1}^{\prime}$ and $\pi_{2}^{\prime}$. Since $\left(\pi_{1}, \pi_{2}\right)$ is the maximal ideal of $A$, it follows that there is a unique extension of $v_{i}$ to $E$, which we denote by $\tilde{v}_{i}$. Let $\omega$ be a discrete valuation of $M$ extending $\tilde{v}_{i}$ for some $i$. Then the restriction of $\omega$ to $L$ is equal to $w_{i}^{j_{i}}$ for some $j_{i}$. Let $E_{i}=F\left(\sqrt[\ell]{\pi_{i}}\right)$ and $M_{i}=L\left(\sqrt[\ell]{\pi_{i}}\right)$. Let $\omega^{\prime}$ and $v_{i}^{\prime}$ be the restrictions of $\omega$ and $\tilde{v}_{i}$ to $M_{i}$ and $E_{i}$ respectively. Suppose that $e\left(w_{i}^{j_{i}} / v_{i}\right)=m$. Then, by $(4.1), e\left(\omega^{\prime} / v_{i}^{\prime}\right)=e\left(w_{i}^{j_{i}} / v_{i}\right) / \ell$ and $e\left(\omega / \tilde{v}_{i}\right) \leq e\left(\omega^{\prime} / v_{i}^{\prime}\right)=e\left(w_{i}^{j_{i}} / v_{i}\right) / \ell$. Hence, by induction hypothesis, for each $i=1,2$, there is a unique extension of $\tilde{v}_{i}$ to $M$. Since $L$ is a subfield of $M$, there is a unique extension of $v_{i}$ to $L$. Hence $L \otimes_{F} F_{\pi_{i}}$ is a field.

The next lemma describes the structure of Galois extensions $L / F$ which are unramified on $A$ except possibly at $\pi_{1}$ and $\pi_{2} \in A$ and totally ramified at $\pi_{2}$.

Lemma 4.4. Let $A$ be a complete regular local ring of dimension 2 with residue field $\kappa$ and fraction field $F$. Let $L / F$ be a Galois extension of degree $n$ where $n$ is coprime to $\operatorname{char}(\kappa)$. Let $\mathfrak{m}=\left(\pi_{1}, \pi_{2}\right) \subset A$ be the maximal ideal of $A$. Suppose that $L / F$ is unramified on $A$ except possibly at $\pi_{1}$ and $\pi_{2} \in A$ and totally ramified at $\pi_{2}$. Then $L=F\left(\sqrt[n]{u \pi_{1}^{m} \pi_{2}}\right)$ for some $u \in A$ a unit and some integer $m$.

Proof. Let $G$ be the Galois group of $L / F$. Since the degree of $L / F$ is coprime to $\operatorname{char}(\kappa)$ and $L / F$ is unramified on $A$ except possibly at $\pi_{1}$ and $\pi_{2}$, by $(4.3), L \otimes_{F} F_{\pi_{2}}$ is a field. Since $L \otimes_{F} F_{\pi_{2}} / F_{\pi_{2}}$ is a totally tamely ramified extension, $F_{\pi_{2}}$ contains a primitive $n^{\text {th }}$ root of unity and we have $L \otimes_{F} F_{\pi_{2}}=F_{\pi_{2}}\left(\sqrt[n]{\theta \pi_{2}}\right)$ for some $\theta \in F_{\pi_{2}}$ which is a unit in the discrete valuation ring of $F_{\pi_{2}}$ by $([2$, Proposition $1, \mathrm{p}-32])$. In particular $G$ is a cyclic group. Since $F_{\pi_{2}}$ contains a primitive $n^{\text {th }}$ root of unity, the residue field $\kappa\left(\pi_{2}\right)$ of $F_{\pi_{2}}$ contains a primitive $n^{\text {th }}$ root of unity. Since $\kappa$ is the residue field of $\kappa\left(\pi_{2}\right), \kappa$ also contains a primitive $n^{\text {th }}$ root of unity. Since $A$ is complete, by Hensel's lemma, $F$ contains a primitive $n^{\text {th }}$ root of unity.

Hence $L=F(\sqrt[n]{a})$ for some $a \in F$. Since $L / F$ is unramified on $A$ except possibly at $\pi_{1}, \pi_{2}$, we can choose $a=u \pi_{1}^{m} \pi_{2}^{d}$ for some $u \in A$ a unit and integers $m, d$. Since $L / F$ is totally ramified at $\pi_{2}, d$ is coprime to $n$ and hence we can assume that $d=1$.

Next we consider Galois extensions $L / F$ which are unramified on $A$ except possibly at $\pi_{1}$.

Lemma 4.5. Let $A$ be a complete regular local ring of dimension 2 with residue field $\kappa$ and fraction field $F$. Let $L / F$ be a Galois extension of degree coprime to char $(\kappa)$. Let $\mathfrak{m}=\left(\pi_{1}, \pi_{2}\right) \subset A$ be the maximal ideal of $A$. Suppose that $L / F$ is unramified on $A$ except possibly at $\pi_{1}$. Then there exists a subextension $L_{1} / F$ of $L / F$ such that:

- $L_{1} / F$ is unramified on $A$, and 
- $L=L_{1}\left(\sqrt[e]{u \pi_{1}}\right)$ for some unit $u$ in the integral closure of $A$ in $L_{1}$.

Proof. Let $G$ be the Galois group of $L / F$. Since $L \otimes_{F} F_{\pi_{1}} / F_{\pi_{1}}$ is a field extension (4.3), the Galois group $\operatorname{Gal}\left(L \otimes_{F} F_{\pi_{1}} / F_{\pi_{1}}\right)$ is isomorphic to $G$. We will identify these two groups. We consider the inertia group $H$ of the extension $L \otimes_{F} F_{\pi_{1}} / F_{\pi_{1}}$ which is a subgroup of $G$. Let $L_{1}=L^{H}$. Then $\left(L \otimes_{F} F_{\pi_{1}}\right)^{H}=L_{1} \otimes_{F} F_{\pi_{1}}$ is unramified over $F_{\pi_{1}}$ by $\left(\left[2\right.\right.$, Theorem 2, p-27]). Hence $\left(L_{1}\right)_{\pi_{1}} \cong L_{1} \otimes_{F} F_{\pi_{1}}$ is unramified over $F_{\pi_{1}}$ and $L_{1} / F$ is unramified at $\pi_{1}$. Since $L / F$ is unramified on $A$ except possibly at $\pi_{1}, L_{1} / F$ is unramified on $A$. Then the integral closure $B$ of $A$ in $L_{1}$ is a regular local ring with maximal ideal $\left(\pi_{1}, \pi_{2}\right)$. Let $e=\left[L: L_{1}\right]$. Since $L / F$ is unramified on $A$ except possibly at $\pi_{1}, L / L_{1}$ is unramified on $B$ except possibly at $\pi_{1}$. Hence by (4.4), with the roles of $\pi_{1}$ and $\pi_{2}$ interchanged, we have $L=L_{1}\left(\sqrt[e]{u \pi_{2}^{m} \pi_{1}}\right)$ for some $u \in B$ a unit. Since $L / L_{1}$ is unramified on $B$ except possibly at $\pi_{1}, m$ is divisibly by $e$ and hence $L=L_{1}\left(\sqrt[e]{u \pi_{1}}\right)$.

The next theorem describes the structure of Galois extensions $L / F$ unramified on $A$ except possibly at $\pi_{1}$ and $\pi_{2} \in A$, hence generalising (4.4) and (4.5).

Theorem 4.6. Let $A$ be a complete regular local ring of dimension 2 with residue field $\kappa$ and fraction field $F$. Let $L / F$ be a Galois extension of degree coprime to $\operatorname{char}(\kappa)$. Let $\mathfrak{m}=\left(\pi_{1}, \pi_{2}\right) \subset A$ be the maximal ideal of $A$. Suppose that $L / F$ is unramified on $A$ except possibly at $\pi_{1}$ and $\pi_{2} \in A$. Then there exists subfields $L_{1}$ and $L_{2}$ of $L$ such that:

- $F \subseteq L_{1} \subseteq L_{2} \subseteq L$,

- $L_{1} / F$ is unramified on $A$,

- $L_{2}=L_{1}\left(\sqrt[d_{1}]{u \pi_{1}}\right)$ for some unit $u$ in the integral closure of $A$ in $L_{1}$, and

- $L=L_{2}\left(\sqrt[d_{2}]{v\left(\sqrt[d_{1}]{u \pi_{1}}\right)^{i} \pi_{2}}\right)$ for some unit $v$ in the integral closure of $A$ in $L_{2}$.

Proof. Let $G$ be the Galois group of $L / F$. Since $L \otimes_{F} F_{\pi_{2}} / F_{\pi_{2}}$ is a field extension (4.3), the Galois group $\operatorname{Gal}\left(L \otimes_{F} F_{\pi_{2}} / F_{\pi_{2}}\right)$ is isomorphic to $G$. We identify these two groups. We consider the inertia group $H$ of the extension $L \otimes_{F} F_{\pi_{2}} / F_{\pi_{2}}$ which is a subgroup of $G$. Let $L_{2}=L^{H}$. Then, as in (4.5), $L_{2} / F$ is unramified on $A$ except possibly at $\pi_{1}$. Hence, by (4.5), there exists a sub extension $L_{1} / F$ of $L_{2} / F$ such that $L_{1} / F$ is unramified on $A$ and $L_{2}=L_{1}\left(\sqrt[d_{1}]{u \pi_{1}}\right)$ for some unit $u$ in the integral closure of $A$ in $L_{1}$. Let $B$ be the integral closure of $A$ in $L_{2}$. Then $B$ is a regular local ring with maximal ideal $\left(\sqrt[d_{1}]{u \pi_{1}}, \pi_{2}\right)$ by $\left(\left[16\right.\right.$, Lemma 3.2.]). Since $L / L_{2}$ is unramified on $B$ except possibly at $\sqrt[d_{1}]{u \pi_{1}}, \pi_{2}$ and totally ramified at $\pi_{2}$, by (4.4), $L=L_{2}\left(\sqrt[d_{2}]{v\left(\sqrt[d_{1}]{u \pi_{1}}\right)^{i} \pi_{2}}\right)$ for some unit $v \in B$.

4.2. Norms over two dimensional complete fields. Let $A, F, \pi_{1}, \pi_{2}$ be as in 4.2. Let $\lambda=u \pi_{1}^{r} \pi_{2}^{s}$ for some unit $u \in A$ and integers $r, s$. In this subsection, we show that if $\lambda$ is a norm from the extension $L \otimes_{F} F_{\pi_{1}} / F_{\pi_{1}}$, then $\lambda$ is a norm from the extension $L / F$.

We begin by describing the elements of $F_{\pi_{i}}^{\times} / F_{\pi_{i}}^{\times n}$ : 
Lemma 4.7. Let $A$ be a complete regular local ring of dimension 2 with residue field $\kappa$ and fraction field $F$. Let $\mathfrak{m}=\left(\pi_{1}, \pi_{2}\right)$ be the maximal ideal of $A$. Let $m \geq 1$ be an integer coprime to char $(\kappa)$. Let $F_{\pi_{1}}$ be the completion of $F$ at the discrete valuation of $F$ given by $\pi_{1}$. Then every element in $F_{\pi_{1}}$ can be written as $u \pi_{1}^{s} \pi_{2}^{t} a^{m}$ for some $u \in A$ a unit, $a \in F_{\pi_{1}}$ and integers $s, t$.

Proof. Let $\widehat{A}_{\left(\pi_{1}\right)}$ be the completion of the local ring $A_{\left(\pi_{1}\right)}$. Then $F_{\pi_{1}}$ is the fraction field of $\widehat{A}_{\left(\pi_{1}\right)}$. Let $x \in F_{\pi_{1}}$. Then $x=v \pi_{1}{ }^{s}$ for some unit $v \in \widehat{A}_{\left(\pi_{1}\right)}$ and integer $s$. Let $\bar{v}$ be the image of $v$ in the residue field $\kappa\left(\pi_{1}\right)$ of $F_{\pi_{1}}$. Since $\kappa\left(\pi_{1}\right)$ is the fraction field of $A /\left(\pi_{1}\right)$ and $A /\left(\pi_{1}\right)$ is a discrete valuation ring with the image $\bar{\pi}_{2}$ as a parameter, we can write $\bar{v}=z{\overline{\pi_{2}}}^{t}$ for some unit $z \in A /\left(\pi_{1}\right)$ and integer $t$. Let $u \in A$ with $\bar{u}=z \in A /\left(\pi_{1}\right)$. Since $z$ is a unit in $A /\left(\pi_{1}\right), u$ is a unit in $A$. Hence $x^{-1} u \pi_{1}^{s} \pi_{2}^{t}$ maps to 1 in $\kappa\left(\pi_{1}\right)$. Since $m$ is coprime to char $(\kappa)$, by Hensel's lemma, $x=u \pi_{1}{ }^{s} \pi_{2}{ }^{t} a^{m}$ for some $a \in F_{\pi_{1}}$.

Lemma 4.8. Let $A$ be a complete regular local ring of dimension 2 with residue field $\kappa$ and fraction field $F$. Let $L / F$ be a Galois field extension of degree $n$ where $n$ is coprime to char $(\kappa)$. Let $\mathfrak{m}=\left(\pi_{1}, \pi_{2}\right)$ be the maximal ideal of $A$. Suppose that $L / F$ is unramified on $A$ except possibly at $\pi_{1}$. Let $\lambda=u \pi_{1}^{r} \pi_{2}^{s}$ for some unit $u \in A$ and integers $r$, s. If $\lambda$ is a norm from the extension $L \otimes_{F} F_{\pi_{1}} / F_{\pi_{1}}$, then $\lambda$ is a norm from the extension $L / F$.

Proof. Let $\mu \in L \otimes_{F} F_{\pi_{1}}$ be such that $N_{L \otimes_{F} F_{\pi_{1}} / F_{\pi_{1}}}(\mu)=\lambda$.

Since $L / F$ is a Galois extension which is unramified on $A$ except possibly at $\pi_{1}$, by (4.5), we have a subfield $F \subseteq L_{1} \subseteq L$ such that $L_{1} / F$ is unramified on $A$ and $L=L_{1}\left(\sqrt[e]{v \pi_{1}}\right)$ where $e=\left[L: L_{1}\right]$ and $v$ is a unit in the integral closure of $A$ in $L_{1}$. Let $B$ be the integral closure of $A$ in $L$. Then $B$ is a regular local ring with maximal ideal $\left(\sqrt[e]{v \pi_{1}}, \pi_{2}\right)$ by $([16$, Lemma 3.2.]). Hence, by $(4.7), \mu=$ $w \sqrt[e]{v \pi_{1}} i \pi_{2}^{j} b^{n}$ for some integers $i, j, b \in L \otimes_{F} F_{\pi_{1}}$ and $w$ a unit in $B$. Let $\theta=$ $w \sqrt[e]{v \pi_{1}} i \pi_{2}^{j} \in L$. Since $N_{L \otimes_{F}} F_{\pi_{1}} / F_{\pi_{1}}(\mu)=\lambda$, we have $N_{L / F}\left(\theta^{-1}\right) \lambda=N_{L \otimes_{F} F_{\pi_{1}} / F_{\pi_{1}}}\left(b^{n}\right) \in$ $F_{\pi_{1}}^{\times n}$. Since $N_{L / F}\left(\theta^{-1}\right) \lambda=\left[N_{L / F}(w) v \pi_{1}^{\frac{n}{e} i} \pi_{2}^{n j}\right]^{-1} u \pi_{1}^{r} \pi_{2}^{s}=\left[u\left(N_{L / F}(w)\right)^{-1}\right] \pi_{1}^{r-\frac{n}{e} i} \pi_{2}^{s-n j}$ and $u\left(N_{L / F}(w)\right)^{-1}$ is a unit in $A$, by $\left(\left[17\right.\right.$, Corollary 5.5.]), $N_{L / F}\left(\theta^{-1}\right) \lambda \in F^{\times n}$. In particular $N_{L / F}\left(\theta^{-1}\right) \lambda$ is a norm from the extension $L / F$ and hence $\lambda$ is a norm from $L / F$.

Theorem 4.9. Let $A$ be a complete regular local ring of dimension 2 with residue field $\kappa$ and fraction field $F$. Let $L / F$ be a Galois field extension of degree $n$ where $n$ is coprime to char $(\kappa)$. Let $\mathfrak{m}=\left(\pi_{1}, \pi_{2}\right)$ be the maximal ideal of $A$. Suppose that $L / F$ is unramified on $A$ except possibly at $\pi_{1}, \pi_{2}$. Let $\lambda=u \pi_{1}^{r} \pi_{2}^{s}$ for some unit $u \in A$ and integers $r$, s. If $\lambda$ is a norm from the extension $L \otimes_{F} F_{\pi_{1}} / F_{\pi_{1}}$, then $\lambda$ is a norm from the extension $L / F$.

Proof. Let $\mu \in L \otimes_{F} F_{\pi_{1}}$ be such that $N_{L \otimes_{F} F_{\pi_{1}} / F_{\pi_{1}}}(\mu)=\lambda$. We show by induction on the degree of the field extension $L / F$ that $\lambda$ is a norm from the extension $L / F$.

Since $L / F$ is a Galois extension which is unramified on $A$ except possibly at $\pi_{1}$ and $\pi_{2}$, we have subfields $L_{1}$ and $L_{2}$ as in (4.6).

Let $B$ be the integral closure of $A$ in $L_{2}$. Then $B$ is a complete regular local ring with maximal ideal $\left(\sqrt[d_{1}]{v \pi_{1}}, \pi_{2}\right)$ by $([16$, Lemma 3.2.]). By (4.7), we have $N_{L \otimes_{F} F_{\pi_{1}} / L_{2} \otimes_{F} F_{\pi_{1}}}(\mu)=w \sqrt[d_{1}]{v \pi_{1}} i \pi_{2}^{j} b^{n}$ for some integers $i, j, b \in L_{2} \otimes_{F} F_{\pi_{1}}$ and $w$ a unit in $B$. Then $\theta=w \sqrt[d_{1}]{v \pi_{1}} i_{2}^{j}$ is a norm from $L \otimes_{F} F_{\pi_{1}} / L_{2} \otimes_{F} F_{\pi_{1}}$. 
Suppose that $F \neq L_{2}$. Then $\left[L: L_{2}\right]<[L: F]$ and by induction, $\theta$ is a norm from $L / L_{2}$. Write $\theta=N_{L / L_{2}}\left(\theta^{\prime}\right)$. Then

$$
\begin{aligned}
\lambda & =N_{L \otimes_{F} F_{\pi_{1}} / F_{\pi_{1}}}(\mu) \\
& =N_{L_{2} \otimes_{F} F_{\pi_{1}} / F_{\pi_{1}}}\left(N_{L \otimes_{F} F_{\pi_{1}} / L_{2} \otimes_{F} F_{\pi_{1}}}(\mu)\right) \\
& =N_{L_{2} \otimes_{F} F_{\pi_{1}} / F_{\pi_{1}}}\left(\theta b^{n}\right) \\
& =N_{L_{2} \otimes_{F} F_{\pi_{1}} / F_{\pi_{1}}}(\theta) N_{L_{2} \otimes_{F} F_{\pi_{1}} / F_{\pi_{1}}}\left(b^{n}\right) \\
& =N_{L_{2} / F}(\theta) N_{L_{2} \otimes_{F} F_{\pi_{1}} / F_{\pi_{1}}}(b)^{n} \\
& =N_{L_{2} / F}\left(N_{L / L_{2}}\left(\theta^{\prime}\right) N_{L_{2} \otimes_{F} F_{\pi_{1}} / F_{\pi_{1}}}(b)^{n}\right. \\
& =N_{L / F}\left(\theta^{\prime}\right) N_{L_{2} \otimes_{F} F_{\pi_{1}} / F_{\pi_{1}}}(b)^{n}
\end{aligned}
$$

Since $N_{L / F}\left(\theta^{\prime}\right)=N_{L_{2} / F}(\theta)=N_{L_{2} / F}\left(w \sqrt[d_{1}]{v \pi_{1}} \pi_{1}^{j}\right), N_{L / F}\left(\theta^{\prime}\right)^{-1} \lambda$ is a product of a unit in $A$ with a power of $\pi_{1}$ and a power of $\pi_{2}$. Since $N_{L / F}\left(\theta^{\prime}\right)^{-1} \lambda=N_{L_{2} \otimes_{F} F_{\pi_{1}} / F_{\pi_{1}}}(b)^{n} \in$ $F_{\pi_{1}}^{n}$, by ([17, Corollary 5.5.]), we conclude that $N_{L / F}\left(\theta^{\prime}\right)^{-1} \lambda$ is a $n^{\text {th }}$ power in $F$ and hence a norm from $L$ to $F$. Hence $\lambda$ is also a norm from $L$ to $F$.

Now suppose $F=L_{2}$. Then $L=F\left(\sqrt[n]{v \pi_{2}}\right)$ where $v$ is a unit in $A$ and hence $L / F$ is a cyclic extension of degree $n$. Let $\sigma$ be a generator of the Galois group of $L / F$ and $C$ be the cyclic algebra $(L, \sigma, \lambda)$. Since $L / F$ is unramified on $A$ except at $\pi_{2}, C$ is a unramified on $A$ except possibly at $\pi_{1}$ and $\pi_{2}$. Since $\lambda$ is a norm from $L \otimes_{F} F_{\pi_{1}}$, $C \otimes_{F} F_{\pi_{1}}$ is a split algebra. Thus, by ([17, Corollary 5.5.]), $C$ is a split algebra and hence $\lambda$ is a norm from the extension $L / F$ by ([1, Theorem $6, \mathrm{p}-95])$.

\section{5. Ш vs $\amalg_{X}$}

In this section, we compare the groups $\amalg_{X}\left(F, T_{L / F}\right)$ and $\amalg\left(F, T_{L / F}\right)$ for a semiglobal field $F$ and a finite Galois extension $L / F$ of degree coprime to the characteristic of the residue field. The proof uses Theorem 4.9 from the previous section. This allows us to use patching techniques to prove the local-global principle for norm one tori $T_{L / F}$ over $F$ with respect to discrete valuations.

Theorem 5.1. Let $K$ be a complete discretely valued field with residue field $\kappa$. Let $F$ be the function field of a smooth, projective, geometrically integral curve over $K$ and $\mathscr{X}_{0}$ a regular proper model of $F$ with reduced special fibre $X_{0}$. Let $L / F$ be a Galois field extension of degree coprime to char $(\kappa)$. Then $\amalg\left(F, T_{L / F}\right)=\bigcup_{X} \amalg_{X}\left(F, T_{L / F}\right)$, where $X$ is running over the reduced special fibres of regular proper models $\mathscr{X}$ of $F$ which are obtained as a sequence of blow-ups of $\mathscr{X}_{0}$ centered at closed points of $\mathscr{X}_{0}$.

Proof. Let $x \in \amalg\left(F, T_{L / F}\right) \subseteq H^{1}\left(F, T_{L / F}\right)$. Since $H^{1}\left(F, T_{L / F}\right) \simeq F^{\times} / N_{L / F}\left(L^{\times}\right)$, let $\lambda \in F^{\times}$be a lift of $x$. For a regular proper model $\mathscr{X}$ of $F$, let $\operatorname{supp}_{\mathscr{X}}(\lambda)$ denote the support of $\lambda$ in $\mathscr{X}$ and $\operatorname{ram}_{\mathscr{X}}(L / F)$ denote the ramification locus for the extension $L / F$ with respect to $\mathscr{X}$. By $([13, \mathrm{p}-193])$, there exists a sequence of blow-ups $\mathscr{X} \rightarrow$ $\mathscr{X}_{0}$ centered at closed points of $\mathscr{X}_{0}$ such that the union of $\operatorname{supp}_{\mathscr{X}}(\lambda), \operatorname{ram}_{\mathscr{X}}(L / F)$ and the reduced special fibre $X$ of $\mathscr{X}$ is a union of regular curves with normal crossings. We show that $x \in \amalg_{X}\left(F, T_{L / F}\right)$.

Let $P \in X$. First suppose $P$ is a generic point of $X$. Then $P$ gives a discrete valuation $\nu$ of $F$ with $F_{\nu}=F_{P}$. Since $x \in \amalg\left(F, T_{L / F}\right), x$ maps to 0 in $H^{1}\left(F_{P}, T_{L / F}\right)$.

Next suppose that $P$ is a closed point. Let $\eta_{1}$ be the generic point of an irreducible component of $X$ containing $P$. Let $\mathcal{O}_{\mathscr{X}, P}$ be the local ring at $P$ and $\mathfrak{m}_{\mathscr{X}, P}$ be its maximal ideal. Then, by our choice of $\mathscr{X}, \mathfrak{m}_{\mathscr{X}, P}=\left(\pi_{1}, \pi_{2}\right)$ where $\pi_{1}$ is a prime 
defining $\eta_{1}$ at $P, \lambda=u \pi_{1}^{r} \pi_{2}^{s}$ for some unit $u \in \mathcal{O}_{\mathscr{X}, P}$ and integers $r, s$, and $L \otimes_{F} F_{P} / F_{P}$ is unramified on $\mathcal{O}_{\mathscr{X}, P}$ except possibly at $\pi_{1}, \pi_{2}$. Since $L / F$ is a Galois extension, $L \otimes_{F} F_{P}=\prod L_{P}$ for some Galois extension $L_{P} / F_{P}$. Since $L \otimes_{F} F_{P} / F_{P}$ is unramified on $\mathcal{O}_{\mathscr{X}, P}$ except possibly at $\pi_{1}, \pi_{2}, L_{P} / F_{P}$ is unramified on $\mathcal{O}_{\mathscr{X}, P}$ except possibly at $\pi_{1}, \pi_{2}$. Since $\lambda$ is a lift of $x \in \amalg_{X}\left(F, T_{L / F}\right), \lambda$ is a norm from $L \otimes_{F} F_{\eta_{1}} / F_{\eta_{1}}$. Since $F_{\eta_{1}} \subset F_{P, \eta_{1}}, \lambda$ is a norm from $L \otimes_{F} F_{P, \eta_{1}} / F_{P, \eta_{1}}$. Hence $\lambda$ is a norm from $L_{P} \otimes_{F_{P}} F_{P, \eta_{1}} / F_{P, \eta_{1}}$. Thus, by (4.9), $\lambda$ is a norm from $L_{P} / F_{P}$ and $x$ maps to 0 in $H^{1}\left(F_{P}, T_{L / F}\right)$. Therefore $x \in \amalg_{X}\left(F, T_{L / F}\right)$. By ([12, Proposition 8.2.]), we have

$\bigcup_{X} \amalg_{X}\left(F, T_{L / F}\right)=\amalg\left(F, T_{L / F}\right)$, where $X$ is running over the reduced special fibres of regular proper models $\mathscr{X}$ of $F$ which are obtained as a sequence of blow-ups of $\mathscr{X}_{0}$ centered at closed points of $\mathscr{X}_{0}$.

Remark 5.2. The proof of (5.1) also works if we just consider divisorial discrete valuations instead of considering all discrete valuations on $F$.

\section{Local Global Principle}

In this section, we prove the local-global principle for norm one tori over semiglobal fields with respect to points on the reduced special fibre under some assumptions on the semi-global field and the residue field. This, combined with Theorem 5.1, allows us to conclude that under the same assumptions, the local-global principle for norm one tori also holds with respect to discrete valuations.

Lemma 6.1. Let $K$ be a complete discretely valued field with residue field $\kappa$ and $F$ be the function field of a smooth, projective, geometrically integral curve over $K$. Let $\mathscr{X}$ be a regular proper model of $F$ with the reduced special fibre $X$ a union of regular curves with normal crossings. Let $L / F$ be a Galois extension over $F$ of degree $n$. Let $P \in X$ be a closed point and $U$ an irreducible open subset of $X$ with $P$ in the closure of $U$. Suppose that:

- $n$ is coprime to char $(\kappa)$,

- $K$ contains a primitive $n^{\text {th }}$ root of unity $\rho$, and

- for all finite Galois extensions $l / \kappa(P)$ of degree d dividing $n$,

$$
\begin{array}{r}
T_{l / \kappa(P)}(\kappa(P))=R T_{l / \kappa(P)}(\kappa(P))<\rho^{\frac{n}{d}}>. \\
\text { Then } T_{L \otimes_{F} F_{P, U} / F_{P, U}}\left(F_{P, U}\right)=R T_{L \otimes_{F} F_{P, U} / F_{P, U}}\left(F_{P, U}\right)<\rho>.
\end{array}
$$

Proof. Let $\kappa(U)$ be the function field of $U$. Since $X$ is a union of regular curves, $P$ gives a discrete valuation on $\kappa(U)$. Let $\kappa(U)_{P}$ be the completion of $\kappa(U)$ at $P$. Then, by definition, $F_{P, U}$ is a complete discretely valued field with residue field $\kappa(U)_{P}$. Since $L / F$ is a Galois extension of degree $n, L \otimes_{F} F_{P, U} \simeq \prod L_{0}$ for some finite Galois extension $L_{0} / F_{P, U}$ of degree $d$ dividing $n$. Since $F_{P, U}$ is a complete discretely valued field with residue field $\kappa(U)_{P}, L_{0}$ is a complete discretely valued field with residue field $M_{0}$ a finite extension of $\kappa(U)_{P}$ of degree $d_{1}$ dividing $d$. Since $\kappa(U)_{P}$ is a complete discretely valued field with residue field $\kappa(P), M_{0}$ is a complete discretely valued field with residue field $l_{0}$ a finite Galois extension of $\kappa(P)$ of degree $d_{2}$ dividing $d_{1}$. Hence, 
by the assumption on $\kappa(P)$ and (3.2), we have

$$
T_{M_{0} / \kappa(U)_{P}}\left(\kappa(U)_{P}\right)=R T_{M_{0} / \kappa(U)_{P}}\left(\kappa(U)_{P}\right)<\rho^{\frac{n}{d_{1}}}>\text {. }
$$

Hence, once again by (3.2), we have

$$
T_{L_{0} / F_{P, U}}\left(F_{P, U}\right)=R T_{L_{0} / F_{P, U}}\left(F_{P, U}\right)<\rho^{n / d}>.
$$

Since $L \otimes_{F} F_{P, U}$ is the product of $\frac{n}{d}$ copies of $L_{0}$, by (2.6), we have

$$
T_{L \otimes_{F} F_{P, U} / F_{P, U}}\left(F_{P, U}\right)=R T_{L \otimes_{F} F_{P, U} / F_{P, U}}\left(F_{P, U}\right)<\rho>.
$$

We are now ready to prove the local-global principle for norm one tori over semiglobal fields with respect to points on the reduced special fibre of the model under some assumptions on the given semi-global field $F$ and the residue field $\kappa$.

Theorem 6.2. Let $K$ be a complete discretely valued field with residue field $\kappa$ and $F$ be the function field of a smooth, projective, geometrically integral curve over $K$. Let $\mathscr{X}$ be a regular proper model of $F$ with reduced special fibre $X$ a union of regular curves with normal crossings. Let $L / F$ be a Galois extension over $F$ of degree $n$. Suppose

- $n$ is coprime to $\operatorname{char}(\kappa)$,

- $K$ contains a primitive $n^{\text {th }}$ root of unity $\rho$,

- for all finite extensions $\kappa^{\prime} / \kappa$ and for all finite Galois extensions $l / \kappa^{\prime}$ of degree $d$ dividing $n$,

$$
T_{l / \kappa^{\prime}}\left(\kappa^{\prime}\right)=R T_{l / \kappa^{\prime}}\left(\kappa^{\prime}\right)<\rho^{\frac{n}{d}}>
$$

- the graph associated to $\mathscr{X}$ is a tree.

Then $\amalg_{X}\left(F, T_{L / F}\right)=0$.

Proof. Let $\mathcal{P}$ be a finite set of closed points of $X$ containing all the nodal points of $X$. By $\left(\left[12\right.\right.$, Corollary 5.9.]), it is enough to show that $\amalg_{\mathcal{P}}\left(F, T_{L / F}\right)=0$. Let $X \backslash \mathcal{P}=\cup_{i} U_{i}$. Then each $U_{i}$ is an irreducible open subset of $X$. By ([12, Corollary 3.6.]), it is enough to show that the product map

$$
\psi: \prod_{i} T_{L / F}\left(F_{U_{i}}\right) \times \prod_{P \in \mathcal{P}} T_{L / F}\left(F_{P}\right) \rightarrow \prod_{\left(P, U_{i}\right)} T_{L / F}\left(F_{P, U_{i}}\right)
$$

is onto, where the product on the right hand side is taken over all pairs $\left(P, U_{i}\right)$ with $P \in \mathcal{P}$ and $U_{i}$ such that $P$ in the closure of $U_{i}$.

Let $\left(\lambda_{P, U_{i}}\right) \in \prod_{\left(P, U_{i}\right)} T_{L / F}\left(F_{P, U_{i}}\right)$. We show that $\left(\lambda_{P, U_{i}}\right)$ is in the image of $\psi$. By (6.1), for each pair $\left(P, U_{i}\right)$ with $P$ in the closure of $U_{i}$, we have $\lambda_{P, U_{i}}=\rho^{j_{P, U_{i}}} \mu_{P, U_{i}}$ for some integer $j_{P, U_{i}}$ and $\mu_{P, U_{i}} \in R T_{L / F}\left(F_{P, U_{i}}\right)$. Let $G$ be the Galois group of $L / F$. For each $\sigma \in G$, there exists $a_{\sigma, P, U_{i}} \in\left(L \otimes_{F} F_{P, U_{i}}\right)^{\times}$such that

$$
\mu_{P, U_{i}}=\prod_{\sigma \in G(L / F)} \sigma\left(a_{\sigma, P, U_{i}}\right)\left(a_{\sigma, P, U_{i}}\right)^{-1}
$$


Since the group $R_{L / F}\left(\mathbb{G}_{m}\right)$ is $F$-rational, by ([11, Theorem 3.6.]),

$$
\prod_{i}\left(L \otimes_{F} F_{U_{i}}\right)^{\times} \times \prod_{P \in \mathcal{P}}\left(L \otimes_{F} F_{P}\right)^{\times} \rightarrow \prod_{\left(P, U_{i}\right)}\left(L \otimes_{F} F_{P, U_{i}}\right)^{\times}
$$

is onto. Hence for each $\sigma \in G$, there exist $b_{\sigma, U_{i}} \in\left(L \otimes_{F} F_{U_{i}}\right)^{\times}$and $b_{\sigma, P} \in\left(L \otimes_{F} F_{P}\right)^{\times}$ such that $a_{\sigma, P, U_{i}}=b_{\sigma, U_{i}} b_{\sigma, P}$. We have

$$
\begin{aligned}
\mu_{P, U_{i}} & =\prod_{\sigma \in G(L / F)} \sigma\left(a_{\sigma, P, U_{i}}\right)\left(a_{\sigma, P, U_{i}}\right)^{-1} \\
& =\prod_{\sigma \in G(L / F)} \sigma\left(b_{\sigma, U_{i}} b_{\sigma, P}\right)\left(b_{\sigma, U_{i}} b_{\sigma, P}\right)^{-1} \\
& =\prod_{\sigma \in G(L / F)} \sigma\left(b_{\sigma, U_{i}}\right)\left(b_{\sigma, U_{i}}\right)^{-1} \sigma\left(b_{\sigma, P}\right)\left(b_{\sigma, P}\right)^{-1}
\end{aligned}
$$

Since $\sigma\left(b_{\sigma, U_{i}}\right)\left(b_{\sigma, U_{i}}\right)^{-1} \in T_{L / F}\left(F_{U_{i}}\right)$ and $\sigma\left(b_{\sigma, P}\right)\left(b_{\sigma, P}\right)^{-1} \in T_{L / F}\left(F_{P}\right),\left(\mu_{P, U_{i}}\right)$ is in the image of $\psi$.

Since $T_{L / F}(F)$ is a group and $\rho \in T_{L / F}(F)$, by $(2.3),\left(\rho^{j_{P, U_{i}}}\right)$ is in the image of $\psi$. Since $\psi$ is a homomorphism, $\left(\lambda_{P, U_{i}}\right)$ is in the image of $\psi$, hence proving that $\psi$ is onto.

Using the above theorem and Theorem 5.1, we get that the under same assumptions, we have the local-global principle for norm one tori over semi-global fields with respect to discrete valuations:

Theorem 6.3. With the hypothesis as in Theorem 6.2, we have $\amalg\left(F, T_{L / F}\right)=0$.

Proof. Let $\mathscr{X}$ be a regular proper model of $F$ which is obtained as a sequence of blowups of $\mathscr{X}_{0}$ at closed points. Since the graph $\Gamma\left(\mathscr{X}_{0}\right)$ is a tree, $\Gamma(\mathscr{X})$ is also a tree $([12$, Remark 6.1(b)]). Let $X$ be the reduced special fibre of $\mathscr{X}$. Then $\amalg_{X}\left(F, T_{L / F}\right)=0$ (6.2). Thus, by (5.1), we have $\amalg\left(F, T_{L / F}\right)=0$.

Corollary 6.4. Let $K$ be an $m$-local field with first residue field $\kappa$ or an iterated Laurent series in $m$ variables over an algebraically closed field $\kappa$. Let $F$ be the function field of a smooth, projective, geometrically integral curve over $K$ and $L / F$ be a finite Galois extension of degree $n$ with $(n, \operatorname{char}(\kappa))=1$. Let $\mathscr{X}$ be a regular proper model of $F$ with reduced special fibre $X$ a union of regular curves with normal crossings. Suppose that the graph associated to $\mathscr{X}$ is a tree. If $K$ contains a primitive $n^{\text {th }}$ root of unity, then $\amalg\left(F, T_{L / F}\right)=0$.

Proof. Follows from (3.4) and (6.3).

Remark 6.5. By (5.2), the results also hold true if we just consider divisorial discrete valuations instead of all discrete valuations on $F$ in (6.3) and (6.4).

\section{Counterexamples}

Let $K$ be a complete discretely valued field with residue field algebraically closed. Colliot-Thélène, Parimala and Suresh ([5, Section 3.1. \& Proposition 5.9.]) constructed a function field of a smooth, projective, geometrically integral curve over $K$ and a Galois extension $L / F$ with Galois group $\mathbf{Z} / 2 \mathbf{Z} \times \mathbf{Z} / 2 \mathbf{Z}$ such that the localglobal principle fails for the norm one torus $T_{L / F}$ associated to $L / F$. They use higher reciprocity laws to detect non-trivial elements in $\amalg\left(F, T_{L / F}\right)$. In this section, 
we produce examples of Galois extensions $L / F$ with Galois group $\mathbf{Z} / n \mathbf{Z} \times \mathbf{Z} / n \mathbf{Z}$ and using patching techniques, we show that $\amalg\left(F, T_{L / F}\right)$ is non-trivial.

Let $k$ be a number field and $L_{1}, L_{2}$ be two Galois extensions of $k$. Let $T$ be the $k$-torus given by $N_{L_{1} / k}\left(z_{1}\right) N_{L_{2} / k}\left(z_{2}\right)=1$. If $L_{1}$ and $L_{2}$ are linearly disjoint, then Demarche and Wei ([6, Theorem 1]) proved that the local-global principle holds for $T$. In this section, we also give an example to show that a similar result does not hold in general for function fields of curves over a complete discretely valued field.

Proposition 7.1. Let $A$ be a unique factorization domain and $F$ be its fraction field. Let $L / F$ be a finite Galois extension and $B$ be the integral closure of $A$ in $L$. Suppose that $B$ is a unique factorization domain. Then every element in $T_{L / F}(F)$ can be written as $s \theta$ for some $s \in R T_{L / F}(L)$ and $\theta \in B$ a unit.

Proof. Let $\lambda \in T_{L / F}(F)$. Then $\lambda \in L^{\times}$and $N_{L / F}(\lambda)=1$. Since $L$ is the fraction field of $B, \lambda=\frac{\alpha}{\beta}$ for some $\alpha, \beta \in B$. Since $N_{L / F}(\lambda)=1, N_{L / F}(\alpha)=N_{L / F}(\beta)$. Let $p \in B$ be a prime. Since $A$ is a unique factorization domain, $p B \cap A=q A$ for some prime $q \in A$ and $N_{L / F}(p)=v q^{r}$ for some unit $v \in A$. Suppose that $p$ divides $\alpha$ in $B$. Then $N_{L / F}(p)$ divides $N_{L / F}(\alpha)$ in $A$ and hence $q$ divides $N_{L / F}(\alpha)$. Since $N_{L / F}(\alpha)=$ $N_{L / F}(\beta)$, there exists a prime $p^{\prime} \in B$ such that $p^{\prime}$ divides $\beta$ and $p^{\prime} B \cap A=q A$. Since $L / F$ is a Galois extension, there exists $\sigma \in \operatorname{Gal}(L / F)$ such that $p=w \sigma\left(p^{\prime}\right)$ for some unit $w \in B$. Write $\alpha=p \alpha^{\prime}$ and $\beta=p^{\prime} \beta^{\prime}$. Then $\lambda=\frac{\alpha}{\beta}=\frac{p}{p^{\prime}} \frac{\alpha^{\prime}}{\beta^{\prime}}=\frac{\sigma p^{\prime}}{p^{\prime}} \frac{w \alpha^{\prime}}{\beta^{\prime}}$. Since $B$ is a unique factorization domain, the proposition follows by induction on the number of prime factors of $\alpha$ in $B$.

Proposition 7.2. Let $A$ be a complete regular local ring of dimension 2 with maximal ideal $(\pi, \delta)$, fraction field $F$ and residue field $\kappa$. Let $n$ be a positive integer which is coprime to $\operatorname{char}(\kappa)$. Let $L=F(\sqrt[n]{\pi}, \sqrt[n]{\delta})$. Suppose that $F$ contains a primitive $n^{2}-$ th root of unity $\rho$. Then $T_{L / F}(F)=R T_{L / F}(F)<\rho>$.

Proof. Let $B$ be the integral closure of $A$ in $L$. Then $B$ is a regular local ring of dimension 2 with fraction field $L$ and residue field $\kappa$ ([16, Corollary 3.3.]). Let $\lambda \in$ $T_{L / F}(F)$. Then $\lambda \in L^{\times}$with $N_{L / F}(\lambda)=1$. Then, by (7.1), there exists $s \in R T_{L / F}(F)$ and a unit $\theta \in B$ such that $\lambda=s \theta$. Since the residue fields of $A$ and $B$ are equal, there exists $\theta_{1} \in A$ such that $\theta \equiv \theta_{1}$ modulo the maximal ideal of $B$. Since $n$ is coprime to $\operatorname{char}(\kappa)$, by Hensel's lemma, we have $\theta=\theta_{1} \alpha^{n^{2}}$ for some unit $\alpha \in B$. Let $s_{1}=$ $N_{L / F}(\alpha)^{-1} \alpha^{n^{2}} \in L$. Then, by (2.7), $s_{1} \in R T_{L / F}(F)$. Let $a=\theta_{1} N_{L / F}(\alpha) \in F$. Then $\theta=a s_{1}$. Thus $\lambda=s \theta=s a s_{1}=s s_{1} a$. Since $N_{L / F}(\lambda)=1=N_{L / F}(s)=N_{L / F}\left(s_{1}\right)$, $1=N_{L / F}(a)=a^{n^{2}}$. Thus $a \in<\rho>$. Hence $\lambda=s s_{1} a \in R T_{L / F}(F)<\rho>$.

Lemma 7.3. Let $F$ be a complete discretely valued field with residue field $\kappa$ and ring of integers $R$. Let $n$ be a positive integer coprime to char $(\kappa)$. Let $\pi \in R$ be a parameter and $u \in R$ a unit with $[F(\sqrt[n]{u}): F]=n$. Let $L=F(\sqrt[n]{u}, \sqrt[n]{\pi})$. Suppose that $F$ contains a primitive $n^{2}$-th root of unity $\rho$. Then $\rho^{t} \in R T_{L / F}(F)$ if and only if $n$ divides $t$.

Proof. Let $\sigma$ be the automorphism of $L / F$ given by $\sigma(\sqrt[n]{\pi})=\rho \sqrt[n]{\pi}$ and $\sigma(\sqrt[n]{u})=$ $\sqrt[n]{u}$ and $\tau$ be the automorphism $L / F$ given by $\tau(\sqrt[n]{u})=\rho \sqrt[n]{u}$ and $\tau(\sqrt[n]{\pi})=\sqrt[n]{\pi}$. Then the Galois group of $L / F$ is an abelian group of order $n^{2}$ generated by $\sigma$ and $\tau$ and hence $R T_{L / F}(F)$ is generated by the set $\left\{\frac{\sigma(a)}{a} \frac{\tau(b)}{b} \mid a, b \in L^{\times}\right\}$. Since $\rho^{n}=$ $\tau(\sqrt[n]{u}) / \sqrt[n]{u} \in R T_{L / F}(F), \rho^{n j} \in R T_{L / F}(F)$ for any integer $j$. 
Conversely, suppose $\rho^{t} \in R T_{L / F}(F)$ for some integer $t$. Without loss of generality, we may assume that $1 \leq t \leq n^{2}$. Then $\rho^{t}=a^{-1} \sigma(a) b^{-1} \tau(b)$ for some $a, b \in L$. Let $L^{\prime}=F(\sqrt[n]{\pi})$. Since $\rho \in F$ and $N_{L / L^{\prime}}\left(b^{-1} \tau(b)\right)=1$, we have $\rho^{n t}=$ $N_{L / L^{\prime}}(a)^{-1} N_{L / L^{\prime}}(\sigma(a))$. Let $c=N_{L / L^{\prime}}(a) \in L^{\prime}$. Since $\sigma(c)=\sigma\left(N_{L / L^{\prime}}(a)\right)=N_{L / L^{\prime}}(\sigma(a))$, we have $\sigma(c)=\rho^{n t} c$. Hence $\sigma\left(c^{n}\right)=(\sigma(c))^{n}=\left(\rho^{n t}\right)^{n} c^{n}=c^{n}$. Since $L^{\prime} / F$ is a Galois extension with Galois group generated by $\sigma, c^{n} \in F$. Thus $c=\theta \sqrt[n]{\pi}^{m}$ for some integer $m$ and $\theta \in F$. Since $L / L^{\prime}$ is an unramified extension of degree $n$ and $c$ is a norm from $L / L^{\prime}$, the valuation of $c$ is divisible by $n$. Since $\theta \in F$ and $\sqrt[n]{\pi}$ is a parameter in $L^{\prime}, m=n r$ for some $r$. Hence $c \in F$ and $\rho^{n t}=c^{-1} \sigma(c)=1$. Since $\rho$ is a primitive $n^{2}$-th root of unity, $n$ divides $t$.

Notation 7.4. Let $A$ be a semi-local regular ring of dimension 2 with three maximal ideals $m_{1}, m_{2}, m_{3}$. Suppose that there exist three prime elements $\pi_{1}, \pi_{2}, \pi_{3} \in A$ such that $m_{1}=\left(\pi_{2}, \pi_{3}\right), m_{2}=\left(\pi_{1}, \pi_{3}\right)$ and $m_{3}=\left(\pi_{1}, \pi_{2}\right)$. Suppose that $\pi_{i} \notin m_{i}$ for all $i$. Let $n \geq 2$ be an integer coprime to $\operatorname{char}\left(A / m_{i}\right)$ for all $i$. Let $F$ be the fraction field of $A$. For $1 \leq i \leq 3$, let $\widehat{A}_{m_{i}}$ be the completion of $A$ at $m_{i}, F_{m_{i}}$ be the fraction field $\widehat{A}_{m_{i}}$ and $F_{\pi_{j}}$ be the completion of $F$ at the discrete valuation given by $\pi_{j}$. Let $1 \leq i \neq j, k \leq 3$. Since $m_{i}=\left(\pi_{j}, \pi_{k}\right), \widehat{A}_{m_{i}}$ is a regular local ring with maximal ideal $\left(\pi_{j}, \pi_{k}\right)$. In particular, $\pi_{j}$ gives a discrete valuation on $F_{m_{i}}$ which extends the discrete valuation on $F$ given by $\pi_{j}$. Let $F_{m_{i}, \pi_{j}}$ be the completion of $F_{m_{i}}$ at the discrete valuation given by $\pi_{j}$. Then $F_{\pi_{j}} \subset F_{m_{i}, \pi_{j}}$. Let $L=F\left(\sqrt[n]{\pi_{1} \pi_{2}}, \sqrt[n]{\pi_{2} \pi_{3}}\right)$. Suppose that $F$ contains $\rho$, a primitive $n^{2}$-th root of unity.

Corollary 7.5. With notations as in (7.4), we have $T_{L / F}\left(F_{m_{i}}\right)=R T_{L / F}\left(F_{m_{i}}\right)<$ $\rho>$.

Proof. Since $\pi_{2}$ is a unit at $m_{2}$, we have $m_{2} A_{m_{2}}=\left(\pi_{1} \pi_{2}, \pi_{3} \pi_{2}\right)$. Hence, by $(7.2)$, we have $T_{L / F}\left(F_{m_{2}}\right)=R T_{L / F}\left(F_{m_{2}}\right)<\rho>$. Since $\pi_{1}$ is a unit at $m_{1}, m_{1} A_{m_{1}}=$ $\left(\pi_{1} \pi_{2}, \pi_{1}^{-1} \pi_{3}\right)$. Since $L=F\left(\sqrt[n]{\pi_{1} \pi_{2}}, \sqrt[n]{\pi_{2} \pi_{3}}\right)=F\left(\sqrt[n]{\pi_{1} \pi_{2}}, \sqrt[n]{\pi_{1}^{-1} \pi_{3}}\right)$, by $(7.2)$, we have $T_{L / F}\left(F_{m_{1}}\right)=R T_{L / F}\left(F_{m_{1}}\right)<\rho>$. Similarly, $T_{L / F}\left(F_{m_{3}}\right)=R T_{L / F}\left(F_{m_{3}}\right)<\rho>$.

Corollary 7.6. With notations as in (7.4), we have $T_{L / F}\left(F_{\pi_{i}}\right)=R T_{L / F}\left(F_{\pi_{i}}\right)<\rho>$.

Proof. Let $\kappa\left(\nu_{\pi_{i}}\right)$ be the residue field of $F_{\pi_{i}}$. The discrete valuation $\nu_{\pi_{i}}$ of $F$ given by $\pi_{i}$ has unique extension $\tilde{\nu}_{\pi_{i}}$ to $L$. Since $F$ contains a primitive $n^{\text {th }}$ root of unity, the residue field $\kappa\left(\tilde{\nu}_{\pi_{i}}\right)$ of $L$ at $\tilde{\nu}_{\pi_{i}}$ is a cyclic extension of $\kappa(\pi)$ of degree $n$. In particular, $T_{\kappa\left(\tilde{\nu}_{\pi_{i}}\right) / \kappa\left(\nu_{\pi_{i}}\right)}\left(\kappa\left(\nu_{\pi_{i}}\right)\right)=R T_{\kappa\left(\tilde{\nu}_{\pi_{i}}\right) / \kappa\left(\nu_{\pi_{i}}\right)}\left(\kappa\left(\nu_{\pi_{i}}\right)\right)$. Hence, by $(3.2)$,

$$
T_{L / F}\left(F_{\pi_{i}}\right)=R T_{L / F}\left(F_{\pi_{i}}\right)<\rho>.
$$

Corollary 7.7. Let $F_{m_{i}, \pi_{j}}$ be as in (7.4). Then $\rho^{t} \in R T_{L / F}\left(F_{m_{i}, \pi_{j}}\right)$ if and only if $n$ divides $t$.

Proof. Since the residue field of $F_{m_{i}, \pi_{j}}$ is a complete discretely valued field with the image of $\pi_{k}(k \neq i, j)$ as a parameter and the image of $\pi_{i}$ as a unit, it is easy to see that $L \otimes_{F} F_{m_{i}, \pi_{j}} \simeq F_{m_{i}, \pi_{j}}\left(\sqrt[n]{v \pi_{j}}, \sqrt[n]{u}\right)$ for some units $u$ and $v$ such that $\left[F_{m_{i}, \pi_{j}}(\sqrt[n]{u}): F_{m_{i}, \pi_{j}}\right]=n$. Thus, the corollary follows from (7.3).

For each $1 \leq i \neq j \leq 3$, we have inclusions fields $F_{m_{i}} \rightarrow F_{m_{i}, \pi_{j}}$ and $F_{\pi_{j}} \rightarrow F_{m_{i}, \pi_{j}}$. Thus we have the induced homororphisms $\alpha_{i j}: T_{L / F}\left(F_{m_{i}}\right) / R \rightarrow T_{L / F}\left(F_{m_{i}, \pi_{j}}\right) / R$ and $\beta_{j i}: T_{L / F}\left(F_{\pi_{j}}\right) / R \rightarrow T_{L / F}\left(F_{m_{i}, \pi_{j}}\right) / R$. 
Lemma 7.8. The product map

$$
\phi:\left(\prod_{i=1}^{3} T_{L / F}\left(F_{m_{i}}\right) / R\right) \times\left(\prod_{j=1}^{3} T_{L / F}\left(F_{\pi_{j}}\right) / R\right) \rightarrow \prod_{1 \leq i \neq j \leq 3}\left(T_{L / F}\left(F_{m_{i}, \pi_{j}}\right) / R\right)
$$

is not onto.

Proof. Let $y_{12}=\rho \in T_{L / F}\left(F_{m_{1}, \pi_{2}}\right)$ and $y_{i j}=1 \in T_{L / F}\left(F_{m_{i}, \pi_{j}}\right)$ for all $i \neq j$ and $(i, j) \neq(1,2)$. Then we show that $y=\left(y_{i j}\right) \in \prod_{1 \leq i \neq j \leq 3}\left(T_{L / F}\left(F_{m_{i}, \pi_{j}}\right) / R\right)$ is not in the image of $\phi$.

Suppose $y$ is in the image of $\phi$. Then there exist $a_{i} \in T_{L / F}\left(F_{m_{i}}\right)$ and $b_{j} \in$ $T_{L / F}\left(F_{\pi_{j}}\right)$ such that $\phi\left(a_{1}, a_{2}, a_{3}, b_{1}, b_{2}, b_{3}\right)=y$ modulo $R$-trivial elements. Then we have $\alpha_{12}\left(a_{1}\right) \beta_{21}\left(b_{2}\right)=y_{12}=\rho$ modulo $R$-trivial elements and $\alpha_{i j}\left(a_{i}\right) \beta_{j i}\left(b_{j}\right) \in$ $R T_{L / F}\left(F_{m_{i}, \pi_{j}}\right)$ for all $i \neq j$ and $(i, j) \neq(1,2)$. By $(7.5)$ and $(7.6)$, we have $a_{i}=c_{i} \rho^{s_{i}}$ for some $c_{i} \in R T_{L / F}\left(F_{m_{i}}\right)$ and $b_{j}=d_{j} \rho^{t_{j}}$ for some $d_{i} \in R T_{L / F}\left(F_{\pi_{j}}\right)$. Hence $a_{i}=\rho^{s_{i}}$ and $b_{j}=\rho^{t_{j}}$ modulo $R$-trivial elements. Since $\rho \in F, \alpha_{i j}(\rho)=\rho$ and $\beta_{j i}(\rho)=\rho$ for all $i \neq j$. We have $\rho=y_{12}=\alpha_{12}\left(a_{1}\right) \beta_{21}\left(b_{2}\right)=\rho^{s_{1}+t_{2}}$ modulo $R$-trivial elements. Hence, by (7.7), $n$ divides $1-s_{1}-t_{2}$.

Let $1 \leq i \neq j \leq 3$ with $(i, j) \neq(1,2)$. Then $1=\alpha_{i j}\left(a_{i}\right) \beta_{j i}\left(b_{j}\right)=\rho^{s_{i}+t_{j}}$ modulo $R$-trivial elements. Hence $\rho^{s_{i}+t_{j}} \in R T_{L / F}\left(F_{m_{i}, \pi_{j}}\right)$ and by (7.7), $n$ divides $s_{i}+t_{j}$. Since $n$ divides $s_{2}+t_{1}$ and $s_{3}+t_{1}, n$ divides $s_{3}-s_{2}$. Since $n$ divides $s_{1}+t_{3}$ and $s_{2}+t_{3}$, $n$ divides $s_{1}-s_{2}$. Hence $n$ divides $s_{1}-s_{3}$. Since $n$ divides $s_{3}+t_{2}, n$ divides $s_{1}+t_{2}$, which contradicts the fact that $n$ divides $1-s_{1}-t_{2}$.

Theorem 7.9. Let $K$ be a complete discretely valued field with residue field $\kappa$ and ring of integers $R$. Let $\mathscr{X}$ be a regular integral surface proper over $R$ and $F$ be its fraction field. Let $X$ denote the reduced special fibre of $\mathscr{X}$. Suppose that $X$ is a union of regular curves with normal crossings. Suppose that there exist three three irreducible curves $X_{1}, X_{2}$ and $X_{3}$ regular on $\mathscr{X}$ such that $X_{i} \cap X_{j}, i \neq j$ has exactly one closed point. Let $n \geq 2$ be an integer coprime to char $(\kappa)$. Suppose that $K$ has a primitive $n^{2}$-th root of unity. Then there exists a Galois extension $L / F$ of degree $n^{2}$ with Galois group isomorphic to $\mathbf{Z} / n \mathbf{Z} \times \mathbf{Z} / n \mathbf{Z}$ such that the local-global principle fails for $T_{L / F}$.

Proof. Let $P_{1}, P_{2}$ and $P_{3}$ be the points of $X_{i} \cap X_{j}, i \neq j$. Let $A$ be the semi local ring at $P_{1}, P_{2}$ and $P_{3}$ on $\mathscr{X}$. Then $A$ has three maximal ideals $m_{1}, m_{2}$ and $m_{3}$. Since $\mathscr{X}$ is regular and each $X_{i}$ is regular on $\mathscr{X}$, there exist primes $\pi_{1}, \pi_{2}, \pi_{3} \in A$ such that $m_{i}=\left(\pi_{j}, \pi_{k}\right)$ for all distinct $i, j, k$. Let $L=F\left(\sqrt[n]{\pi_{1} \pi_{2}}, \sqrt[n]{\pi_{2} \pi_{3}}\right)$. Since $K$ contains primitive $n^{\text {th }}$ root of unity, $L / F$ is a Galois extension with Galois group isomorphic to $\mathbf{Z} / n \mathbf{Z} \times \mathbf{Z} / n \mathbf{Z}$. We claim that the local-global principle fails for $T_{L / F}$.

Let $\mathcal{P}$ be a finite set of closed points of $X$ containing all the singular points of $X$. Let $X \backslash \mathcal{P}=\cup U_{i}$, with $U_{i} \subset X_{i}$ for $i=1,2,3$. By ([12, Corollary 3.6.]), it is enough to show that the product map

$$
\prod_{P \in \mathcal{P}} T_{L / F}\left(F_{P}\right) \times \prod_{i} T_{L / F}\left(F_{U_{i}}\right) \rightarrow \prod_{P, U_{i}} T_{L / F}\left(F_{P, U_{i}}\right)
$$


is not onto. Since $X_{1}, X_{2}, X_{3}$ are the only curves in $X$ passing through $P_{1}, P_{2}$ or $P_{3}$, it is enough to show that

$$
\phi: \prod_{i=1}^{3} T_{L / F}\left(F_{P_{i}}\right) \times \prod_{j=1}^{3} T_{L / F}\left(F_{U_{j}}\right) \rightarrow \prod_{P_{i}, U_{j}} T_{L / F}\left(F_{P_{i}, U_{j}}\right)
$$

is not onto. Since $F_{U_{j}} \subset F_{\pi_{j}}$ and $F_{P_{i}, U_{j}}=F_{P_{i}, \pi_{j}}, \phi$ factors as

$$
\prod_{i=1}^{3} T_{L / F}\left(F_{P_{i}}\right) \times \prod_{j=1}^{3} T_{L / F}\left(F_{U_{j}}\right) \rightarrow \prod_{i=1}^{3} T_{L / F}\left(F_{P_{i}}\right) \times \prod_{j=1}^{3} T_{L / F}\left(F_{\pi_{j}}\right) \rightarrow \prod_{P_{i}, \pi_{j}} T_{L / F}\left(F_{P_{i}, \pi_{j}}\right) .
$$

Since, by (7.8),

$$
\prod_{i=1}^{3}\left(T_{L / F}\left(F_{\pi_{i}}\right) / R\right) \times \prod_{j=1}^{3}\left(T_{L / F}\left(F_{P_{j}}\right) / R\right) \rightarrow \prod_{U_{i}, P_{j}}\left(T_{L / F}\left(F_{P_{i}, U_{j}}\right) / R\right)
$$

is not onto, $\phi$ is not onto.

Remark 7.10. The above theorem for $\kappa$ algebraically closed and $n=2$ is proved by Colliot-Thélène, Parimala and Suresh ([5, Section 3.1. \& Corollary 6.2.]).

Corollary 7.11. Let $K$ be a complete discretely valued field with residue field $\kappa$ and ring of integers $R$. Let $t \in R$ be a parameter. Let $\mathscr{X}=\operatorname{Proj}\left(R[x, y, z] /\left\langle x y(x+y-z)-t z^{3}\right\rangle\right)$. Let $X$ be the special fibre of $\mathscr{X}$. Then $X=\operatorname{Proj}(\kappa[x, y, z] /\langle x y(x+y-z)\rangle)$ which is reduced. Then $X$ has three irreducible components $X_{1}, X_{2}, X_{3}$ and $X_{i}$ intersects $X_{j}, i \neq j$ at exactly one point. Let $F$ be the function field of $\mathscr{X}$. Then $F \simeq K(x)[y] /\langle x y(x+y-1)-t\rangle$. Let $n \geq 2$ be coprime to char $(\kappa)$. Suppose that $K$ contains a primitive $n^{2}$-th root of unity. Let $L=F(\sqrt[n]{x y}, \sqrt[n]{y(x-1)})$. Then $L / F$ is a Galois extension with Galois group isomorphic to $\mathbf{Z} / n \mathbf{Z} \times \mathbf{Z} / n \mathbf{Z}$. By (7.9), the local-global principle for fails for $T_{L / F}$.

Proof. Let $U=\operatorname{Spec} R[x, y] /(x y(x+y-1)-t)$. Then $U$ is an affine open subset of $\mathscr{X}$. Let $P_{1}=(1,0), P_{2}=(0,1)$ and $P_{3}=(0,0)$ be the three closed points of $U$. Let $A$ be the semi local ring at $P_{1}, P_{2}$ and $P_{3}$ and let $m_{i}$ be the maximal ideal of $A$ corresponding to $P_{i}$. Then $m_{1}=(x+y-1, y), m_{2}=(x, x+y-1)$ and $m_{3}=(x, y)$. Hence, by (7.9), the local-global principle fails for $T_{L / F}$.

Corollary 7.12. Let $K$ be a complete discretely valued field with residue field $\kappa$ and ring of integers $R$. Let $t \in R$ be a parameter. Let $\mathscr{X}=\operatorname{Proj}\left(R[x, y, z] /\left\langle x y(x+y-z)(x-2 z)-t z^{4}\right\rangle\right)$ and $F$ be the function field of $\mathscr{X}$. Then $F \simeq K(x)[y] /(x y(x+y-1)(x-2)-t)$. Let $\theta_{1}=(x-2) /(x-2+x y(x+y-1))$ and $\theta_{2}=(y-2) /(y-2+x y(x+y-1))$. Let $n \geq 2$ with $6 n$ coprime to $\operatorname{char}(\kappa)$. Let $L_{1}=F(\sqrt[n]{x y}, \sqrt[n]{y(x+y-1)})$ and $L_{2}=F\left(\sqrt[n]{x y \theta_{1}}, \sqrt[n]{y(x+y-1) \theta_{2}}\right)$. Then $L_{1}$ and $L_{2}$ are Galois extensions of $F$ that are linearly disjoint and the local-global principle fails for $T_{L_{1} \times L_{2} / F}$.

Proof. To show that the local-global principle fails for $T_{L_{1} \times L_{2} / F}$, by ([11, Theorem 3.6.]) and as in the proof of (7.9), it is enough to show that

$$
\phi: \prod_{i=1}^{3}\left(T_{L_{1} \times L_{2} / F}\left(F_{\pi_{i}}\right) / R\right) \times \prod_{j=1}^{3}\left(T_{L_{1} \times L_{2} / F}\left(F_{P_{j}}\right) / R\right) \rightarrow \prod_{U_{i}, P_{j}}\left(T_{L_{1} \times L_{2} / F}\left(F_{P_{i}, U_{j}}\right) / R\right)
$$

is not onto. 
Let $U=\operatorname{Spec} R[x, y] /(x y(x+y-1)(x-2)-t)$. Then $U$ is an affine open subset of $\mathscr{X}$. Let $P_{1}=(1,0), P_{2}=(0,1), P_{3}=(0,0)$ and $Q=(2,2)$. Let $A$ be the semi local ring at $P_{1}, P_{2}, P_{3}$ and $Q$. Let $m_{i}$ be the maximal ideals of $A$ corresponding to $P_{i}$ and $m$ be the maximal ideal corresponding to $Q$. Let $\pi_{1}=x, \pi_{2}=y$ and $\pi_{3}=x+y-1$. Then $m_{1}=\left(\pi_{2}, \pi_{3}\right), m_{2}=\left(\pi_{1}, \pi_{3}\right), m_{3}=\left(\pi_{1}, \pi_{2}\right)$. We also have $m=(x-2, y-2)$. Since $2 \neq \operatorname{char}(\kappa), x-2$ and $y-2$ are units at $m_{i}$ and $\theta_{i}=1$ modulo $m_{j}$ and $\pi_{j}$. Since $n$ is coprime to $\operatorname{char}(\kappa), \theta_{i} \in F_{P_{i}}^{n}$ and $\theta_{i} \in F_{\pi_{j}}^{n}$ for all $i$ and $j$. Hence $L_{1} \otimes_{F} F_{\pi_{i}} \simeq L_{2} \otimes_{F} F_{\pi_{i}}$ and $L_{1} \otimes_{F} F_{P_{j}} \simeq L_{2} \otimes_{F} F_{P_{j}}$. By $(2.5)$, we have $T_{L_{1} \times L_{2} / F}\left(F_{\pi_{i}}\right) / R \simeq T_{L_{1} / F}\left(F_{\pi_{i}}\right) / \mathrm{R}$, $T_{L_{1} \times L_{2} / F}\left(F_{P_{j}}\right) / R \simeq T_{L_{1} / F}\left(F_{P_{j}}\right) / R$ and $T_{L_{1} \times L_{2} / F}\left(F_{U_{i}, P_{j}}\right) / R \simeq T_{L_{1} / F}\left(F_{U_{i}, P_{j}}\right) / R$. Since, by $(7.8)$,

$$
\prod_{i=1}^{3}\left(T_{L / F}\left(F_{\pi_{i}}\right) / R\right) \times \prod_{j=1}^{3}\left(T_{L / F}\left(F_{P_{j}}\right) / R\right) \rightarrow \prod_{U_{i}, P_{j}}\left(T_{L / F}\left(F_{P_{i}, U_{j}}\right) / R\right)
$$

is not onto, $\phi$ is not onto. Hence the local-global principle fails for $T_{L / F}$.

Since $\pi_{1} \pi_{2}=x y=4$ modulo $m$ and $\pi_{2} \pi_{3}=6$ modulo $m$, we have $L_{1} \otimes_{F} F_{Q}=$ $F_{Q}(\sqrt[n]{4}, \sqrt[n]{6})$. Since $6 n$ is coprime to $\operatorname{char}(\kappa), L_{1} \otimes_{F} F_{Q} / F_{Q}$ is unramified. Since $x y(x+y-1)=12$ modulo $m, x-2+x y(x+y-1)=12 a^{n}$ for some $a \in F_{Q}$. Similarly, $y-2+x y(x+y+1)=12 b^{n}$ for some $b \in F_{Q}$. Hence $L_{2} \otimes_{F} F_{Q}=$ $F_{Q}(\sqrt[n]{(x-2) / 3}, \sqrt[n]{(y-2) / 2})$. Since the maximal ideal $m=(x-2, y-2)$ and 3,2 are units at $m, L_{1} \otimes_{F} F_{Q}$ and $L_{2} \otimes_{F} F_{Q}$ are linearly disjoint over $F_{Q}$. In particular, $L_{1}$ and $L_{2}$ are linearly disjoint over $F$.

Acknowledgements: The author thanks his advisor Prof. V. Suresh for all his help and guidance during this work. The author is also very thankful to Prof. R. Parimala for several helpful discussions. The author thanks Professors J.-L. ColliotThélène and R. Parimala for going through an earlier version of the manuscript and providing their helpful feedbacks. The author thanks the anonymous referee for the constructive feedback due to which the exposition has improved considerably. The author is partially supported by National Science Foundation grants DMS-1463882 and DMS-1801951. The author thanks Emory University and Laney Graduate School for excellent working conditions.

\section{REFERENCES}

[1] A. Albert, Structure of Algebras, Amer. Math. Soc. Colloq. Publ. Vol. 24, Amer. Math. Soc., Providence, RI, 1961, revised printing.

[2] J.W.S. Cassels and A. Fröhlich, Algebraic Number Theory, Thomson Book Company Inc, Washington, D.C, 1967.

[3] J.-L. Colliot-Thélène, J-J Sansuc, La R-équivalence sur les tores, Annales scientifiques de l'É.N.S. $4^{\mathrm{e}}$ série, tome 10, n⿳02(1977), 175-229.

[4] J.-L. Colliot-Thélène, R. Parimala and V. Suresh, Patching and local-global principles for homogeneous spaces over function fields of p-adic curves, Comment. Math. Helv. 87 (2012), 1011-1033.

[5] J.-L. Colliot-Thélène, R. Parimala and V. Suresh, Lois de réciprocité supérieures et points rationnels, Trans. Amer. Math. Soc. 368 (2016), 4219-4255.

[6] C. Demarche and D. Wei, Hasse principle and weak approximation for multinorm equations, Israel J. Math. 202 (2014), 275-293.

[7] E. Bayer-Fluckiger, T.- Y. Lee and R. Parimala, Hasse principles for multinorm equations (2018), to appear in Advances Math. 
[8] P. Gille, Lectures on R-equivalence on linear algebraic groups, online lecture notes(2010), http://math.univ-lyon1.fr/homes-www/gille/prenotes/grenoble.pdf

[9] Thomas W. Hungerford, Algebra, Graduate Texts in Mathematics v.73, Springer, 2003.

[10] Y. Hu, Hasse Principle for Simply Connected Groups over Function Fields of Surfaces, J. Ramanujan Math. Soc. 29 (2014), no. 2, 155-199.

[11] D. Harbater, J. Hartmann and D. Krashen, Applications of patching to quadratic forms and central simple algebras, Invent. Math. 178 (2009), 231-263.

[12] D. Harbater, J. Hartmann and D. Krashen, Local-global principles for torsors over arithmetic curves, American Journal of Mathematics, 137 (2015), 1559-1612.

[13] J. Lipman, Introduction to resolution of singularities, Proceedings in Pure Mathematics, vol. 29, American Mathematical Society, Providence, RI (1975), pp. 187-230.

[14] S. Lang, Algebra, 3rd edition, Springer(2002).

[15] R. Preeti, Classification theorems for hermitian forms, the Rost kernel and Hasse principle over fields with cd(2) $(k) \leq$ 3, Journal of Algebra 385(2013):294-313.

[16] R. Parimala and V. Suresh, Period-index and u-invariant questions for function fields over complete discretely valued fields, Invent. math. 197 (2014), 215-235.

[17] R. Parimala, R. Preeti and V. Suresh, Local-global principle for reduced norms over function fields of p-adic curves, preprint 2016.

[18] V. Platonov and A. Rapinchuk, Algebraic Groups and Number Theory, Academic Press, 1st edition (1993), v.139.

[19] R. Sharifi, Algebraic Number Theory, online lecture notes, https://www . math.ucla.edu/ sharifi/algnum.pdf.

Department of Mathematics, Emory University, 400 Dowman Drive NE, Atlanta, GA 30322, USA

E-mail address: sumitcmishra@gmail.com 